\title{
Armendariz property for skew PBW extensions and their classical ring of quotients
}

\author{
Armando Reyes ${ }^{a *}$, Héctor SuÁRez ${ }^{b}$ \\ ${ }^{a}$ Universidad Nacional de Colombia, Departamento de Matemáticas, Bogotá, \\ Colombia. \\ ${ }^{b}$ Universidad Pedagógica y Tecnológica de Colombia, Escuela de Matemáticas y \\ Estadística, Tunja, Colombia.
}

\begin{abstract}
We consider a first approach to the notion of Armendariz ring for a skew Poincaré-Birkhoff-Witt (PBW for short) extension, and its classical ring of quotients. As an immediate application of this treatment, we study the properties Baer, quasi-Baer, p.p. and p.q.-Baer rings for these extensions. In this way, we generalize several results in the literature concerning Ore extensions and skew PBW extensions.
\end{abstract}

Keywords: Armendariz, Baer, quasi-Baer, p.p. and p.q-rings, skew PoincaréBirkhoff-Witt extensions.

MSC2010: 16D25, 16E50, 16S36.

\section{Propiedad de Armendariz para las extensiones PBW torcidas y su anillo clásico de cocientes}

Resumen. Consideramos un primer acercamiento a la noción de anillo de Armendariz para una extensión torcida de Poincaré-Birkhoff-Witt (PBW), y su anillo clásico de cocientes. Como una aplicación inmediata de este tratamiento, estudiamos las propiedades de Baer, quasi-Baer, p.p. y p.q.-Baer para estas extensiones. De esta manera, generalizamos varios resultados de la literatura para extensiones de Ore y extensiones PBW torcidas.

Palabras clave: Armendariz, Baer, quasi-Baer, p.p. y p.q.-anillos, extensiones torcidas de Poincaré-Birkhoff-Witt.

\footnotetext{
* E-mail: mareyesv@unal.edu.co

Received: 15 June 2016, Accepted: 04 August 2016.

To cite this article: A. Reyes, H. Suárez, Armendariz property for skew PBW extensions and their classical ring of quotients, Rev. Integr. Temas Mat. 34 (2016), No. 2, 147-168.
} 


\section{Introduction}

In commutative algebra, a ring $B$ is called Armendariz (the term was introduced by Rege and Chhawchharia in [25]) if whenever polynomials $f(x)=a_{0}+a_{1} x+\cdots+a_{n} x^{n}$, $g(x)=b_{0}+b_{1} x+\cdots+b_{m} x^{m} \in B[x]$ satisfy $f(x) g(x)=0$, then $a_{i} b_{j}=0$ for every $i, j$. The interest of this notion lies in its natural and its useful role in understanding the relation between the annihilators of the $\operatorname{ring} B$ and the annihilators of the polynomial ring $B[x]$. In [2], Lemma 1 , Armendariz showed that a reduced ring (i.e., a ring without nonzero nilpotent elements) always satisfies this condition (reduced rings are Abelian -that is, every idempotent is central-, and also semiprime, that is, its prime radical is trivial). With respect to the noncommutative algebra, more exactly the well-known Ore extensions, the notion of Armendariz has been also studied. In this way, commutative and noncommutative treatments have been investigated in several papers (see for example [1], [2], [12], [14], [17], [19], [23], [25] and others).

The examples of noncommutative (and commutative polynomial, of course) rings of interest for us in this article are the skew Poincaré-Birkhoff-Witt extensions introduced in [9]. These structures are more general than Ore extensions of injective type, and contain various well-known groups of algebras such as some types of Auslander-Gorenstein rings, some Calabi-Yau and skew Calabi-Yau algebras, some Artin-Schelter regular algebras, some Koszul algebras, quantum polynomials, some quantum universal enveloping algebras, etc. Indeed, it has been shown that skew PBW extensions contain several algebras which can not be expressed as Ore extensions (universal enveloping algebras of finite Lie algebras, diffusion algebras, and others (see [22], [26], [29], [28], or [30] for more details)).

Precisely, in [28], Remark 3.8, the first author announced one paper with a skew notion of Armendariz ring for skew PBW extensions. Well then, this is the first paper on that promise. Next, we describe the structure of this article. In Section 2 we establish some useful results about skew PBW extensions for the rest of the paper. In Section 3 we introduce two notions of Armendariz for these extensions: $\Sigma$-skew Armendariz (Definition 3.1) and a more general notion, the weak $\Sigma$-skew Armendariz (Definition 3.2). These definitions generalize the cases developed for both classical polynomial rings and Ore extensions of injective type (cf. [1], [2], [12], [14], [17], [19], [23], [24] and [25]). We show that every $\Sigma$-rigid ring ([28], Definition 3.2) is a $\Sigma$-skew Armendariz (Proposition 3.4), but the converse is false as Remark 3.5 shows. In other words, we have the relations $\Sigma$-rigid rings $\varsubsetneqq \Sigma$-skew Armendariz rings $\varsubsetneqq$ weak $\Sigma$-skew Armendariz rings. Nevertheless, in Theorem 3.6 we prove the following equivalences: for a skew PBW extension $A$ of a ring $R, R$ is reduced and $\Sigma$-skew Armendariz $\Leftrightarrow R$ is $\Sigma$-rigid $\Leftrightarrow A$ is reduced. In this way, our Theorem 3.6 generalizes [23], Theorem A, and [7], Theorem 1 and Corollary 3 . In this section, we also present some key results with the aim of proving that if $R$ is a weak $\Sigma$-skew Armendariz ring, then $R$ and $A$ are Abelian (Proposition 3.9 and Corollary 3.10 , respectively). In Section 4, we characterize the weak $\Sigma$-skew Armendariz property over $Q(R)$-the classical ring of quotients of $R$ - in terms of the weak $\Sigma$-skew Armendariz property over $R$. This characterization is formulated in Theorem 4.2, and it turns out a generalization of [24], Theorem 2.3, and [17], Theorem 16. More generally than the characterization presented in Theorem 3.6, our Theorem 4.3 establishes the equivalence between the notions of $\Sigma$-rigid, $\Sigma$-skew Armendariz and weak $\Sigma$-skew Armendariz for the classical ring of quotients of a semiprime Goldie ring $R, Q(R)$, generalizing [24], Corollary 2.5. 
Since $\Sigma$-rigid rings are contained strictly in weak $\Sigma$-skew Armendariz rings, in the last section of the paper, Section 5, we investigate the properties of being Baer, quasi-Baer, p.p. and p.q.-Baer (the interest of studying these properties comes from the question about annihilators of elements, as a key point to compute the uniform -also known as Goldie- dimension for these extensions (see [29]), for skew PBW extensions over weak $\Sigma$ skew Armendariz rings). In this way, our Theorems 5.1 and 5.3 generalizes [28], Theorems 3.9 and 3.12, respectively, and our Corollary 5.5 generalizes [28], Theorems 3.10 and 3.13 . The techniques used here follow some ideas presentes in several articles on Ore extensions, and the results presented are new for skew PBW extensions and generalize others existing in the literature concerning Ore extensions of injective type and skew PBW extensions.

\section{Definitions and elementary properties}

In this section we recall the definition of skew PBW extension and present some key properties of these rings.

Definition 2.1 ([9], Definition 1). Let $R$ and $A$ be rings. We say that $A$ is a skew $P B W$ extension of $R$ (also called a $\sigma-P B W$ extension of $R$ ) if the following conditions hold:

(i) $R \subseteq A$.

(ii) there exist elements $x_{1}, \ldots, x_{n} \in A$ such that $A$ is a left free $R$-module, with basis the basic elements $\operatorname{Mon}(A):=\left\{x^{\alpha}=x_{1}^{\alpha_{1}} \cdots x_{n}^{\alpha_{n}} \mid \alpha=\left(\alpha_{1}, \ldots, \alpha_{n}\right) \in \mathbb{N}^{n}\right\}$, and $x_{1}^{0} \cdots x_{n}^{0}:=1 \in \operatorname{Mon}(A)$.

(iii) For each $1 \leq i \leq n$ and any $r \in R \backslash\{0\}$, there exists an element $c_{i, r} \in R \backslash\{0\}$ such that $x_{i} r-c_{i, r} x_{i} \in R$.

(iv) For any elements $1 \leq i, j \leq n$ there exists $c_{i, j} \in R \backslash\{0\}$ such that $x_{j} x_{i}-c_{i, j} x_{i} x_{j} \in$ $R+R x_{1}+\cdots+R x_{n}$.

Under these conditions we will write $A:=\sigma(R)\left\langle x_{1}, \ldots, x_{n}\right\rangle$.

Proposition 2.2 ([9], Proposition 3). Let $A$ be a skew PBW extension of $R$. For each $1 \leq i \leq n$, there exists an injective endomorphism $\sigma_{i}: R \rightarrow R$ and a $\sigma_{i}$-derivation $\delta_{i}: R \rightarrow R$ such that $x_{i} r=\sigma_{i}(r) x_{i}+\delta_{i}(r)$, for each $r \in R$. We write $\Sigma:=\left\{\sigma_{1}, \ldots, \sigma_{n}\right\}$, and $\Delta:=\left\{\delta_{1}, \ldots, \delta_{n}\right\}$, that is, $\Delta$ is the family of $\Sigma$-derivations in $A$.

Remark 2.3. With respect to the Definition 2.1 and the Proposition 2.2, we have the following facts:

(i) Since $\operatorname{Mon}(A)$ is a left $R$-basis of $A$, the elements $c_{i, r}$ and $c_{i, j}$ in Definition 2.1 are unique.

(ii) In Definition 2.1 (iv), $c_{i, i}=1$. This follows from $x_{i}^{2}-c_{i, i} x_{i}^{2}=s_{0}+s_{1} x_{1}+\cdots+s_{n} x_{n}$, with $s_{i} \in R$, which implies $1-c_{i, i}=0=s_{i}$.

(iii) If $i<j$ and $d_{i}^{\prime}, b_{j}^{\prime} \in R$, then $d_{i}^{\prime} x_{i} b_{j}^{\prime} x_{j}=d_{i}^{\prime}\left[\sigma_{i}\left(b_{j}^{\prime}\right) x_{i}+\delta_{i}\left(b_{j}^{\prime}\right)\right] x_{j}=d_{i}^{\prime} \sigma_{i}\left(b_{j}^{\prime}\right) x_{i} x_{j}+$ $d_{i}^{\prime} \delta_{i}\left(b_{j}^{\prime}\right) x_{j}$. Since $x_{j} x_{i}=c_{i, j} x_{i} x_{j}+r^{(i, j)}+\sum_{k=1}^{n} r_{k}^{(i, j)} x_{k}$, then $d_{j}^{\prime} x_{j} b_{i}^{\prime} x_{i}=$

Vol. 34, No. 2, 2016] 


$$
\begin{aligned}
d_{j}^{\prime}\left[\sigma_{j}\left(b_{i}^{\prime}\right) x_{j}+\delta_{j}\left(b_{i}^{\prime}\right)\right] x_{i}= & d_{j}^{\prime} \sigma_{j}\left(b_{i}^{\prime}\right) x_{j} x_{i}+d_{j}^{\prime} \delta_{j}\left(b_{i}^{\prime}\right) x_{i}=d_{j}^{\prime} \sigma_{j}\left(b_{i}^{\prime}\right)\left(c_{i, j} x_{i} x_{j}+r^{(i, j)}+\right. \\
\left.\sum_{k=1}^{n} r_{k}^{(i, j)} x_{k}\right)+d_{j}^{\prime} \delta_{j}( & \left.b_{i}^{\prime}\right) x_{i}, \text { we obtain } \\
d_{i}^{\prime} x_{i} b_{j}^{\prime} x_{j}+d_{j}^{\prime} x_{j} b_{i}^{\prime} x_{i}= & d_{i}^{\prime} \sigma_{i}\left(b_{j}^{\prime}\right) x_{i} x_{j}+d_{i}^{\prime} \delta_{i}\left(b_{j}^{\prime}\right) x_{j} \\
& +d_{j}^{\prime} \sigma_{j}\left(b_{i}^{\prime}\right)\left(c_{i, j} x_{i} x_{j}+r^{(i, j)}+\sum_{k=1}^{n} r_{k}^{(i, j)} x_{k}\right)+d_{j}^{\prime} \delta_{j}\left(b_{i}^{\prime}\right) x_{i} \\
= & {\left[d_{i}^{\prime} \sigma_{i}\left(b_{j}^{\prime}\right)+d_{j}^{\prime} \sigma_{j}\left(b_{i}^{\prime}\right) c_{i, j}\right] x_{i} x_{j}+d_{j}^{\prime} \delta_{j}\left(b_{i}^{\prime}\right) x_{i} } \\
& +d_{i}^{\prime} \delta_{i}\left(b_{j}^{\prime}\right) x_{j}+d_{j}^{\prime} \sigma_{j}\left(b_{i}^{\prime}\right) r^{(i, j)}+d_{j}^{\prime} \sigma_{j}\left(b_{i}^{\prime}\right) \sum_{k=1}^{n} r_{k}^{(i, j)} x_{k} \\
= & {\left[d_{i}^{\prime} \sigma_{i}\left(b_{j}^{\prime}\right)+d_{j}^{\prime} \sigma_{j}\left(b_{i}^{\prime}\right) c_{i, j}\right] x_{i} x_{j}+\left[d_{j}^{\prime} \delta_{j}\left(b_{i}^{\prime}\right)+d_{j}^{\prime} \sigma_{j}\left(b_{i}^{\prime}\right) r_{i}^{(i, j)}\right] x_{i} } \\
& +\left[d_{i}^{\prime} \delta_{i}\left(b_{j}^{\prime}\right)+d_{j}^{\prime} \sigma_{j}\left(b_{i}^{\prime}\right)\right] x_{j}+d_{j}^{\prime} \sigma_{j}\left(b_{i}^{\prime}\right) r^{(i, j)} \\
& +d_{j}^{\prime} \sigma_{j}\left(b_{i}^{\prime}\right) \sum_{k=1, k \neq i, j}^{n} r_{k}^{(i, j)} x_{k} .
\end{aligned}
$$

Definition 2.4. Let $A$ be a skew PBW extension of $R$.

(i) $A$ is called quasi-commutative if the conditions (iii) and (iv) in Definition 2.1 are replaced by (iii'): for each $1 \leq i \leq n$ and all $r \in R \backslash\{0\}$ there exists $c_{i, r} \in R \backslash\{0\}$ such that $x_{i} r=c_{i, r} x_{i}$; (iv'): for any $1 \leq i, j \leq n$ there exists $c_{i, j} \in R \backslash\{0\}$ such that $x_{j} x_{i}=c_{i, j} x_{i} x_{j}$ ([9], Definition 4$)$.

(ii) $A$ is called bijective if $\sigma_{i}$ is bijective for each $1 \leq i \leq n$, and $c_{i, j}$ is invertible for any $1 \leq i<j \leq n$ ([9], Definition 4$)$.

Example 2.5. The class of skew $P B W$ extensions contains various well-known groups of algebras such as some types of Auslander-Gorenstein rings, some Calabi-Yau and skew Calabi-Yau algebras, some Artin-Schelter regular algebras, some Koszul algebras (cf. [30]), quantum polynomials, some quantum universal enveloping algebras, etc. A detailed list of examples of skew PBW extensions is presented in [22], [26] or [27].

Definition 2.6 ([9], Definition 6). Let $A$ be a skew PBW extension of $R$ with endomorphisms $\sigma_{i}, 1 \leq i \leq n$, as in Proposition 2.2 .

(i) For $\alpha=\left(\alpha_{1}, \ldots, \alpha_{n}\right) \in \mathbb{N}^{n}, \sigma^{\alpha}:=\sigma_{1}^{\alpha_{1}} \cdots \sigma_{n}^{\alpha_{n}},|\alpha|:=\alpha_{1}+\cdots+\alpha_{n}$. If $\beta=$ $\left(\beta_{1}, \ldots, \beta_{n}\right) \in \mathbb{N}^{n}$, then $\alpha+\beta:=\left(\alpha_{1}+\beta_{1}, \ldots, \alpha_{n}+\beta_{n}\right)$.

(ii) For $X=x^{\alpha} \in \operatorname{Mon}(A), \exp (X):=\alpha, \operatorname{deg}(X):=|\alpha|$, and $X_{0}:=1$. The symbol $\succeq$ will denote a total order defined on $\operatorname{Mon}(A)$ (a total order on $\left.\mathbb{N}^{n}\right)$. For an element $x^{\alpha} \in \operatorname{Mon}(A), \exp \left(x^{\alpha}\right):=\alpha \in \mathbb{N}^{n}$. If $x^{\alpha} \succeq x^{\beta}$ but $x^{\alpha} \neq x^{\beta}$, we write $x^{\alpha} \succ x^{\beta}$. Every element $f \in A$ can be expressed uniquely as $f=a_{0}+a_{1} X_{1}+\cdots+a_{m} X_{m}$, with $a_{i} \in R$, and $X_{m} \succ \cdots \succ X_{1}$. With this notation, we define $\operatorname{lm}(f):=X_{m}$, the leading monomial of $f ; \operatorname{lc}(f):=a_{m}$, the leading coefficient of $f ; \operatorname{lt}(f):=a_{m} X_{m}$, the leading term of $f ; \exp (f):=\exp \left(X_{m}\right)$, the order of $f$; and $E(f):=\left\{\exp \left(X_{i}\right) \mid 1 \leq\right.$ $i \leq t\}$. Note that $\operatorname{deg}(f):=\max \left\{\operatorname{deg}\left(X_{i}\right)\right\}_{i=1}^{t}$. Finally, if $f=0$, then $\operatorname{lm}(0):=0$, $\operatorname{lc}(0):=0, \operatorname{lt}(0):=0$. We also consider $X \succ 0$ for any $X \in \operatorname{Mon}(A)$. For a detailed description of monomial orders in skew PBW extensions, see [9], Section 3.

[Revista Integración 
Proposition 2.7 ([9], Theorem 7). Let $A$ be a polynomial ring with coefficients in $R$ and the set of variables $\left\{x_{1}, \ldots, x_{n}\right\} . A$ is a skew $P B W$ extension of $R$ if and only if the following conditions are satisfied:

(i) for each $x^{\alpha} \in \operatorname{Mon}(A)$ and every $0 \neq r \in R$, there exist unique elements $r_{\alpha}:=$ $\sigma^{\alpha}(r) \in R \backslash\{0\}, p_{\alpha, r} \in A$, such that $x^{\alpha} r=r_{\alpha} x^{\alpha}+p_{\alpha, r}$, where $p_{\alpha, r}=0$ or $\operatorname{deg}\left(p_{\alpha, r}\right)<|\alpha|$, if $p_{\alpha, r} \neq 0$. If $r$ is left invertible, so is $r_{\alpha}$.

(ii) For each $x^{\alpha}, x^{\beta} \in \operatorname{Mon}(A)$ there exist unique elements $c_{\alpha, \beta} \in R$ and $p_{\alpha, \beta} \in A$ such that $x^{\alpha} x^{\beta}=c_{\alpha, \beta} x^{\alpha+\beta}+p_{\alpha, \beta}$, where $c_{\alpha, \beta}$ is left invertible, $p_{\alpha, \beta}=0$ or $\operatorname{deg}\left(p_{\alpha, \beta}\right)<$ $|\alpha+\beta|$, if $p_{\alpha, \beta} \neq 0$.

Remark 2.8. With respect to the Proposition 2.7, we have two observations:

(i) ([28], Proposition 2.9) If $\alpha:=\left(\alpha_{1}, \ldots, \alpha_{n}\right) \in \mathbb{N}^{n}$ and $r \in R$, then

$$
\begin{aligned}
x^{\alpha} r= & x_{1}^{\alpha_{1}} x_{2}^{\alpha_{2}} \cdots x_{n-1}^{\alpha_{n-1}} x_{n}^{\alpha_{n}} r=x_{1}^{\alpha_{1}} \cdots x_{n-1}^{\alpha_{n-1}}\left(\sum_{j=1}^{\alpha_{n}} x_{n}^{\alpha_{n}-j} \delta_{n}\left(\sigma_{n}^{j-1}(r)\right) x_{n}^{j-1}\right) \\
& +x_{1}^{\alpha_{1}} \cdots x_{n-2}^{\alpha_{n-2}}\left(\sum_{j=1}^{\alpha_{n-1}} x_{n-1}^{\alpha_{n-1}-j} \delta_{n-1}\left(\sigma_{n-1}^{j-1}\left(\sigma_{n}^{\alpha_{n}}(r)\right)\right) x_{n-1}^{j-1}\right) x_{n}^{\alpha_{n}} \\
& +x_{1}^{\alpha_{1}} \cdots x_{n-3}^{\alpha_{n-3}}\left(\sum_{j=1}^{\alpha_{n-2}} x_{n-2}^{\alpha_{n-2}-j} \delta_{n-2}\left(\sigma_{n-2}^{j-1}\left(\sigma_{n-1}^{\alpha_{n-1}}\left(\sigma_{n}^{\alpha_{n}}(r)\right)\right)\right) x_{n-2}^{j-1}\right) x_{n-1}^{\alpha_{n-1}} x_{n}^{\alpha_{n}} \\
& +\cdots+x_{1}^{\alpha_{1}}\left(\sum_{j=1}^{\alpha_{2}} x_{2}^{\alpha_{2}-j} \delta_{2}\left(\sigma_{2}^{j-1}\left(\sigma_{3}^{\alpha_{3}}\left(\sigma_{4}^{\alpha_{4}}\left(\cdots\left(\sigma_{n}^{\alpha_{n}}(r)\right)\right)\right)\right)\right) x_{2}^{j-1}\right) x_{3}^{\alpha_{3}} x_{4}^{\alpha_{4}} \cdots x_{n-1}^{\alpha_{n-1}} x_{n}^{\alpha_{n}} \\
& +\sigma_{1}^{\alpha_{1}}\left(\sigma_{2}^{\alpha_{2}}\left(\cdots\left(\sigma_{n}^{\alpha_{n}}(r)\right)\right)\right) x_{1}^{\alpha_{1}} \cdots x_{n}^{\alpha_{n}}, \quad \sigma_{j}^{0}:=\operatorname{id}_{R} \text { for } 1 \leq j \leq n .
\end{aligned}
$$

(ii) ([28], Remark 2.10) Using (i), it follows that for the product $a_{i} X_{i} b_{j} Y_{j}$, if $X_{i}:=$ $x_{1}^{\alpha_{i 1}} \cdots x_{n}^{\alpha_{i n}}$ and $Y_{j}:=x_{1}^{\beta_{j 1}} \cdots x_{n}^{\beta_{j n}}$, then

$$
\begin{aligned}
& a_{i} X_{i} b_{j} Y_{j}=a_{i} \sigma^{\alpha_{i}}\left(b_{j}\right) x^{\alpha_{i}} x^{\beta_{j}}+a_{i} p_{\alpha_{i 1}, \sigma_{i 2}^{\alpha_{i 2}}\left(\cdots\left(\sigma_{i n}^{\alpha_{i n}}(b)\right)\right)} x_{2}^{\alpha_{i 2}} \cdots x_{n}^{\alpha_{i n}} x^{\beta_{j}}
\end{aligned}
$$

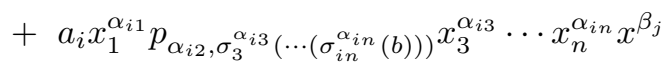

$$
\begin{aligned}
& +a_{i} x_{1}^{\alpha_{i 1}} x_{2}^{\alpha_{i 2}} p_{\alpha_{i 3}, \sigma_{i 4}^{\alpha_{i 4}}\left(\cdots\left(\sigma_{i n}^{\alpha_{i n}}(b)\right)\right)} x_{4}^{\alpha_{i 4}} \cdots x_{n}^{\alpha_{i n}} x^{\beta_{j}} \\
& +\cdots+a_{i} x_{1}^{\alpha_{i 1}} x_{2}^{\alpha_{i 2}} \cdots x_{i(n-2)}^{\alpha_{i(n-2)}} p_{\alpha_{i(n-1)}, \sigma_{i n}^{\alpha_{i n}(b)}} x_{n}^{\alpha_{i n}} x^{\beta_{j}} \\
& +a_{i} x_{1}^{\alpha_{i 1}} \cdots x_{i(n-1)}^{\alpha_{i(n-1)}} p_{\alpha_{i n}, b} x^{\beta_{j}} .
\end{aligned}
$$

In this way, when we compute every summand of $a_{i} X_{i} b_{j} Y_{j}$ we obtain products of the coefficient $a_{i}$ with several evaluations of $b_{j}$ in $\sigma$ 's and $\delta$ 's, depending of the coordinates of $\alpha_{i}$.

\section{3. $\Sigma$-Skew Armendariz and weak $\Sigma$-skew Armendariz}

In [12], p. 104, it was introduced the notion of Armendariz for Ore extensions of endomorphism type (i.e., the $\sigma$-derivation $\delta$ is the zero mapping) in the following way: let $\sigma$

Vol. 34, No. 2, 2016] 
be an endomorphism of a ring $B . B$ is called a skew Armendariz ring with the endomorphism $\sigma$ (simply, a $\sigma$-skew Armendariz ring) if for $p=\sum_{i=0}^{m} a_{i} x^{i}$, and $q=\sum_{j=0}^{n} b_{j} x^{j}$ in $B[x ; \sigma], p q=0$ implies $a_{i} \sigma^{i}\left(b_{j}\right)=0$ for all $0 \leq i \leq m$, and $0 \leq j \leq n$ (note that there exists an endomorphism $\sigma$ of an Armendariz ring $B$ such that $B$ is not $\sigma$-skew Armendariz; see [12], Example 2). In [24], Definition 2.1, it was adapted this definition for general Ore extensions $B[x ; \sigma, \delta]$ with $\delta$ not necessarily the zero mapping. With this in mind, and in the search of a first notion of Armendariz for skew PBW extensions, we introduce the following definition which extends both definitions in [12] and [24].

Definition 3.1. Let $A$ be a skew PBW extension of a ring $R$. $R$ is called a skew Armendariz ring with the family $\Sigma$ (or a $\Sigma$-skew Armendariz ring) if for elements $f=\sum_{i=0}^{m} a_{i} X_{i}$ and $g=\sum_{j=0}^{t} b_{j} Y_{j}$ in $A$, the equality $f g=0$ implies $a_{i} \sigma^{\alpha_{i}}\left(b_{j}\right)=0$ for all $0 \leq i \leq m$ and $0 \leq j \leq t$, where $\alpha_{i}=\exp \left(X_{i}\right)$.

Next we define the weak $\Sigma$-skew Armendariz as a more general class of rings than $\Sigma$-skew Armendariz. Our Definition 3.2 extends [24], Definition 2.2.

Definition 3.2. Let $A$ be a skew PBW extension of a ring $R$. $R$ is called a weak skew Armendariz ring with the family $\Sigma$ (or a weak $\Sigma$-skew Armendariz ring) if for elements $f=\sum_{i=0}^{n} a_{i} x_{i}$ and $g=\sum_{j=0}^{n} b_{j} x_{j}$ in $A\left(x_{0}:=1\right)$, the equality $f g=0$ implies $a_{i} \sigma_{i}\left(b_{j}\right)=0$ for all $0 \leq i, j \leq n\left(\sigma_{0}:=\operatorname{id}_{R}\right)$.

We have the following preliminary result.

Lemma 3.3. If $R$ is a weak skew Armendariz ring, the equality ab $=0$ implies $\sigma^{\alpha}(a) \delta^{\alpha}(b)=\delta^{\alpha}(a) b=0$ for every $a, b \in R$ and $\alpha \in \mathbb{N}^{n}$.

Proof. It is sufficient to prove the case $\sigma_{i}(a) \delta_{i}(b)=\delta_{i}(a) b=0$ for $i=1, \ldots, n$. Since $a b=0$, then $0=\delta_{i}(a b)=\sigma_{i}(a) \delta_{i}(b)+\delta_{i}(a) b$, or equivalently, $\delta_{i}(a) b=-\sigma_{i}(a) \delta_{i}(b)$. Let $f, g \in A$ given by $f=\delta_{i}(a)+0 x_{1}+\cdots+0 x_{i-1}+\sigma_{i}(a) x_{i}+0 x_{i+1}+\cdots+0 x_{n}$, and $g=b+b x_{1}+\cdots+b x_{n}$, respectively. Note that $f g=0$

$$
\begin{aligned}
f g= & \delta_{i}(a) b+\delta_{i}(a) b x_{1}+\cdots+\delta_{i}(a) b x_{n}+\sigma_{i}(a) x_{i} b+\sigma_{i}(a) x_{i} b x_{1}+\cdots+\sigma_{i}(a) x_{i} b x_{n} \\
= & \delta_{i}(a) b+\delta_{i}(a) b x_{1}+\cdots+\delta_{i}(a) b x_{n}+\sigma_{i}(a)\left[\sigma_{i}(b) x_{i}+\delta_{i}(b)\right]+\sigma_{i}(a)\left[\sigma_{i}(b) x_{i}+\delta_{i}(b)\right] x_{1} \\
& +\cdots+\sigma_{i}(a)\left[\sigma_{i}(b) x_{i}+\delta_{i}(b)\right] x_{n} \\
= & \delta_{i}(a) b+\delta_{i}(a) b x_{1}+\cdots+\delta_{i}(a) b x_{n}+\sigma_{i}(a) \sigma_{i}(b) x_{i}+\sigma_{i}(a) \delta_{i}(b)+\sigma_{i}(a) \sigma_{i}(b) x_{i} x_{1} \\
& +\sigma_{i}(a) \delta_{i}(b) x_{1}+\cdots+\sigma_{i}(a) \sigma_{i}(b) x_{i} x_{n}+\sigma_{i}(a) \delta_{i}(b) x_{n} \\
= & 0,
\end{aligned}
$$

since $\delta_{i}(a) b=-\sigma_{i}(a) \delta_{i}(b)$ and $\sigma_{i}(a) \sigma_{i}(b)=\sigma_{i}(a b)=\sigma_{i}(0)=0$. By the weak skew Armendariz condition on $R, \delta_{i}(a) b=0$, and hence, $\sigma_{i}(a) \delta_{i}(b)=0$ for every $i$.

For a ring $B$ with a ring endomorphism $\sigma: B \rightarrow B$, and a $\sigma$-derivation $\delta: B \rightarrow B$, Krempa in [18] considered the Ore extension $B[x ; \sigma, \delta]$ and defined $\sigma$ as a rigid endomorphism if $b \sigma(b)=0$ implies $b=0$ for $b \in B$. Krempa called $B \sigma$-rigid if there exists a rigid endomorphism $\sigma$ of $B$. Since Ore extensions of injective type are particular examples of skew PBW extensions, in [28], Definition 3.2, the first author introduced the following 
definition with the purpose of studying the notion of rigidness for these extensions: if $B$ is a ring and $\Sigma$ is a family of endomorphisms of $B$, then $\Sigma$ is called a rigid endomorphisms family if $r \sigma^{\alpha}(r)=0$ implies $r=0$ for every $r \in B$ and $\alpha \in \mathbb{N}^{n}$. B is called to be $\Sigma$-rigid if there exists a rigid endomorphisms family $\Sigma$ of $B$.

The next proposition shows that every $\Sigma$-rigid ring is an $\Sigma$-skew Armendariz. From now on, we suppose that the elements $c_{i, j}$ in Definition 2.1 (iv) are in the center of $R$.

Proposition 3.4. Let $A$ be a skew $P B W$ extension of a ring $R$. If $R$ is $\Sigma$-rigid, then $R$ is $\Sigma$-skew Armendariz.

Proof. By [28], Proposition 3.6, we know that if $f=\sum_{i=0}^{m} a_{i} X_{i}, g=\sum_{j=0}^{t} b_{j} Y_{j}$ are elements of $A$, then $f g=0$ if and only if $a_{i} b_{j}=0$ for every $0 \leq i \leq m$ and $0 \leq j \leq t$. Now, [28], Lemma 3.3 (i) guarantees that $a_{i} \sigma^{\alpha_{i}}\left(b_{j}\right)=0$ for every $j$, where $\alpha_{i}=\exp \left(X_{i}\right)$, that is, $R$ is $\Sigma$-skew Armendariz.

Remark 3.5. The converse of Proposition 3.4 is false as the following examples show:

- Consider the ring

$$
B=\left\{\left(\begin{array}{ll}
a & t \\
0 & a
\end{array}\right) \mid a \in \mathbb{Z}, t \in \mathbb{Q}\right\}
$$

Then $B$ is a commutative ring. Let $\sigma$ be the automorphism of $R$ given by $\sigma\left(\left(\begin{array}{cc}a & t \\ 0 & a\end{array}\right)\right)=\left(\begin{array}{cc}a & t / 2 \\ 0 & a\end{array}\right)$. In [12], Example 1, it was shown that $R$ is $\sigma$-skew Armendariz and is not a $\sigma$-rigid. Since $\Sigma$-rigid and $\Sigma$-skew Armendariz are generalizations of $\sigma$-rigid and $\sigma$-skew Armendariz, respectively, this example shows that the converse of Proposition 3.4 is false.

- Let $B=\mathbb{Z}_{2}[x]$ be the commutative polynomial ring over $\mathbb{Z}_{2}$, and $\sigma$ the endomorphism of $B=\mathbb{Z}_{2}[x]$ defined by $\sigma(f(x))=f(0)$. Then $B=\mathbb{Z}_{2}[x]$ is $\sigma$-skew Armendariz and is not $\sigma$-rigid ([12], Example 5).

Therefore we have the relations

$$
\Sigma \text {-rigid rings } \varsubsetneqq \Sigma \text {-skew Armendariz rings } \varsubsetneqq \text { weak } \Sigma \text {-skew Armendariz rings. }
$$

In [7] and [23], both authors of those papers give a positive answer to the following question formulated in [12], p. 115: Let $\sigma$ be a monomorphism (or automorphism) of a (commutative) reduced ring $B$ and $B$ be a $\sigma$-skew Armendariz. Is $B \sigma$-rigid? The content of Theorem 3.6 is the generalization of this answer to skew PBW extensions. Again, we suppose that the elements $c_{i, j}$ in Definition 2.1 (iv) commute with every element of $R$.

Theorem 3.6. If $A$ is a skew $P B W$ extension of a ring $R$, then the following statements are equivalent:

(i) $R$ is reduced and $\Sigma$-skew Armendariz;

(ii) $R$ is $\Sigma$-rigid;

(iii) $A$ is reduced.

Vol. 34, No. 2, 2016] 
Proof. (ii) $\Leftrightarrow$ (iii). This equivalence follows from [28], Proposition 3.5. (ii) $\Rightarrow$ (i) From [28] we know that a $\Sigma$-rigid ring is reduced, and as we saw above, every $\Sigma$-rigid ring is also $\Sigma$-skew Armendariz. Let us see (i) $\Rightarrow$ (ii). Suppose that $R$ is reduced, $\Sigma$-skew Armendariz and is not $\Sigma$-rigid. Then there exists $\beta \in \mathbb{N}^{n}$ with $a \sigma^{\beta}(a)=0$ and $a \neq 0$. Note that $\sigma^{\beta}(a) \sigma^{\beta}\left(\sigma^{\beta}(a)\right)=\sigma^{\beta}\left(a \sigma^{\beta}(a)\right)=0$. Using that $R$ is reduced, the equality $\left(\sigma^{\beta}(a) a\right)^{2}=\sigma^{\beta}(a) a \sigma^{\beta}(a) a=0$ implies $\sigma^{\beta}(a) a=0$. Equivalently, since $a \neq 0, \sigma^{\beta}$ is injective, and $R$ is reduced, then $\sigma^{\beta}(a) \neq 0$ and $\left(\sigma^{\beta}(a)\right)^{2} \neq 0$. With this in mind, consider the elements $f=\sigma^{\beta}(a)+\sigma^{\beta}(a) x^{\beta}, g=a-\sigma^{\beta}(a) x^{\beta}$. Then

$$
\begin{aligned}
f g & =\left(\sigma^{\beta}(a)+\sigma^{\beta}(a) x^{\beta}\right)\left(a-\sigma^{\beta}(a) x^{\beta}\right) \\
& =\sigma^{\beta}(a) a-\left(\sigma^{\beta}(a)\right)^{2} x^{\beta}+\sigma^{\beta}(a) x^{\beta} a-\sigma^{\beta}(a) x^{\beta} \sigma^{\beta}(a) x^{\beta} \\
& =-\left(\sigma^{\beta}(a)\right)^{2} x^{\beta}+\sigma^{\beta}(a)\left[\sigma^{\beta}(a) x^{\beta}+p_{\beta, a}\right]-\sigma^{\beta}(a)\left[\sigma^{\beta}\left(\sigma^{\beta}(a)\right) x^{\beta}+q_{\beta, \sigma^{\beta}(a)}\right] x^{\beta} \\
& =\sigma^{\beta}(a) p_{\beta, a}-\sigma^{\beta}\left(a \sigma^{\beta}(a)\right) x^{\beta} x^{\beta}-\sigma^{\beta}(a) q_{\beta, \sigma^{\beta}(a)} x^{\beta} \\
& =\sigma^{\beta}(a) p_{\beta, a}-\sigma^{\beta}(a) q_{\beta, \sigma^{\beta}(a)} x^{\beta},
\end{aligned}
$$

where $p_{\beta, a}=0$ or $\operatorname{deg}\left(p_{\beta, a}\right)<|\beta|$, if $p_{\beta, r} \neq 0$, and $q_{\beta, \sigma^{\beta}(a)}=0$ or $\operatorname{deg}\left(q_{\beta, \sigma^{\beta}(a)}\right)<|\beta|$, if $q_{\beta, \sigma^{\beta}(a)} \neq 0$. Since $a \sigma^{\beta}(a)=\sigma^{\beta}(a) a=0$, Remark 2.8 and Lemma 3.3 guarantee that $\sigma^{\beta}(a) p_{\beta, a}=\sigma^{\beta}(a) q_{\beta, \sigma^{\beta}(a)} x^{\beta}=0$, so $f g=0$. By assumption, $R$ is $\Sigma$-skew Armendariz, that is, $-\left(\sigma^{\beta}(a)\right)^{2}=0$, but $-\left(\sigma^{\beta}(a)\right)^{2} \neq 0$, i.e., we have obtained a contradiction. Hence, $R$ is $\Sigma$-rigid.

Next we present others key results about $\Sigma$-skew Armendariz and weak $\Sigma$-skew Armendariz rings.

Proposition 3.7. If $A$ is a skew $P B W$ extension of a $\Sigma$-skew Armendariz ring $R, e^{2}=$ $e \in A$, with $e=\sum_{i=0}^{m} e_{i} X_{i}$, then $e=e_{0}$.

Proof. Using the equalities $e(1-e)=0=(1-e) e$, i.e., $\left(\sum_{i=0}^{m} e_{i} X_{i}\right)\left(\left(1-e_{0}\right)-\sum_{i=1}^{m} X_{i}\right)=$ 0 and $\left(\left(1-e_{0}\right)-\sum_{i=1}^{m} e_{i} X_{i}\right)\left(\sum_{i=0}^{m} e_{i} X_{i}\right)=0$, and the assumption on $R$, then $e_{0}\left(1-e_{0}\right)=$ $0, e_{0} e_{i}=0$ and $\left(1-e_{0}\right) e_{i}=0$ for $1 \leq i \leq m$. Therefore, $e_{i}=0$ for $1 \leq i \leq m$, that is, $e=e_{0}=e_{0}^{2}$.

Proposition 3.8. If $R$ is a weak $\Sigma$-skew Armendariz ring, and e is an idempotent element of $R$, then $\sigma_{i}(e)=e$ and $\delta_{i}(e)=0$, for every $i=1, \ldots, n$.

Proof. Consider an idempotent element $e$ of $R$. Then $\delta_{i}(e)=\sigma_{i}(e) \delta_{i}(e)+\delta_{i}(e) e$. Let $f, g \in A$ given by $f=\delta_{i}(e)+0 x_{1}+\cdots+0 x_{i-1}+\sigma_{i}(e) x_{i}+0 x_{i+1}+\cdots+0 x_{n}$, and $g=e-1+(e-1) x_{1}+\cdots+(e-1) x_{n}$, respectively. Recall that $\delta_{i}(1)=0$ for every $i$. Let us show that $f g=0$ :

$$
\begin{aligned}
f g= & \delta_{i}(e)(e-1)+\sum_{j=1}^{n} \delta_{i}(e)(e-1) x_{j}+\sigma_{i}(e) x_{i}(e-1)+\sum_{j=1}^{n} \sigma_{i}(e) x_{i}(e-1) x_{j} \\
= & \delta_{i}(e)(e-1)+\sum_{j=1}^{n} \delta_{i}(e)(e-1) x_{j}+\sigma_{i}(e)\left[\sigma_{i}(e-1) x_{i}+\delta_{i}(e-1)\right] \\
& +\sum_{j=1}^{n} \sigma_{i}(e)\left[\sigma_{i}(e-1) x_{i}+\delta_{i}(e-1)\right] x_{j} .
\end{aligned}
$$

[Revista Integración 
Equivalently,

$$
\begin{aligned}
f g= & \delta_{i}(e)(e-1)+\sum_{j=1}^{n} \delta_{i}(e)(e-1) x_{j}+\sigma_{i}(e)\left[\left(\sigma_{i}(e)-\sigma_{i}(1)\right) x_{i}+\delta_{i}(e)\right] \\
& +\sum_{j=1}^{n} \sigma_{i}(e)\left[\left(\sigma_{i}(e)-\sigma_{i}(1)\right) x_{i}+\delta_{i}(e)\right] x_{j} \\
= & \delta_{i}(e)(e-1)+\sum_{j=1}^{n} \delta_{i}(e)(e-1) x_{j}+\sigma_{i}(e)\left[\sigma_{i}(e) x_{i}-x_{i}+\delta_{i}(e)\right] \\
& +\sum_{j=1}^{n} \sigma_{i}(e)\left[\sigma_{i}(e) x_{i}-x_{i}+\delta_{i}(e)\right] x_{j} \\
= & \delta_{i}(e) e-\delta_{i}(e)+\sum_{j=1}^{n}\left(\delta_{i}(e) e-\delta_{i}(e)\right) x_{j}+\sigma_{i}(e) x_{i}-\sigma_{i}(e) x_{i}+\sigma_{i}(e) \delta_{i}(e) \\
& +\sum_{j=1}^{n}\left(\sigma_{i}(e) x_{i}-\sigma_{i}(e) x_{i}+\sigma_{i}(e) \delta_{i}(e)\right) x_{j} \\
= & \delta_{i}(e) e-\delta_{i}(e)+\sum_{j=1}^{n} \delta_{i}(e) e x_{j}-\sum_{j=1}^{n} \delta_{i}(e) x_{j}+\sigma_{i}(e) \delta_{i}(e)+\sum_{j=1}^{n} \sigma_{i}(e) \delta_{i}(e) x_{j} \\
= & \sigma_{i}(e) \delta_{i}(e)+\delta_{i}(e) e-\delta_{i}(e)+\left(\sum_{j=1}^{n}\left(\sigma_{i}(e) \delta_{i}(e)+\delta_{i}(e) e-\delta_{i}(e)\right)\right) x_{j} \\
= & 0 .
\end{aligned}
$$

Since $R$ is weak $\Sigma$-skew Armendariz, we obtain $\delta_{i}(e)(e-1)=0$, i.e., $\delta_{i}(e) e=\delta_{i}(e)$, and hence $\sigma_{i}(e) \delta_{i}(e)=0$.

Now, consider the elements $s$ and $t$ of $A$ given by $s=\delta_{i}(e)-\left(1-\sigma_{i}(e)\right) x_{i}$ and $t=e+\sum_{j=1}^{n} e x_{j}$, respectively. Then $s t=0$. In fact:

$$
\begin{aligned}
s t= & \delta_{i}(e) e+\delta_{i}(e) e \sum_{j=1}^{n} x_{j}-\left(1-\sigma_{i}(e)\right) x_{i} e-\left(1-\sigma_{i}(e)\right) x_{i} e \sum_{j=1}^{n} x_{j} \\
= & \delta_{i}(e) e+\delta_{i}(e) e \sum_{j=1}^{n} x_{j}-x_{i} e+\sigma_{i}(e) x_{i} e-x_{i} e \sum_{j=1}^{n} x_{j}+\sigma_{i}(e) x_{i} e \sum_{j=1}^{n} x_{j} \\
= & \delta_{i}(e) e+\delta_{i}(e) e \sum_{j=1} x_{j}-\left(\sigma_{i}(e) x_{i}+\delta_{i}(e)\right)+\sigma_{i}(e)\left(\sigma_{i}(e) x_{i}+\delta_{i}(e)\right) \\
& -\left(\sigma_{i}(e) x_{i}+\delta_{i}(e)\right) \sum_{j=1}^{n} x_{j}+\sigma_{i}(e)\left(\sigma_{i}(e) x_{i}+\delta_{i}(e)\right) \sum_{j=1}^{n} x_{j} \\
= & \delta_{i}(e) e+\delta_{i}(e) e \sum_{j=1}^{n} x_{j}-\sigma_{i}(e) x_{i}-\delta_{i}(e)+\sigma_{i}(e) x_{i}+\sigma_{i}(e) \delta_{i}(e)-\sigma_{i}(e) x_{i} \sum_{j=1}^{n} x_{j} \\
& -\delta_{i}(e) \sum_{j=1}^{n} x_{j}+\sigma_{i}(e) x_{i} \sum_{j=1}^{n} x_{j}+\sigma_{i}(e) \delta_{i}(e) \sum_{j=1}^{n} x_{j} .
\end{aligned}
$$

Vol. 34, No. 2, 2016] 
Since $\delta_{i}(e)=\delta_{i}(e) e$ and $\sigma_{i}(e) \delta_{i}(e)=0$, then $s t=0$. By Armendariz condition we know that $\delta_{i}(e) e=0$, which shows that $\delta_{i}(e)=0$.

Consider the elements $u, v \in A$ given by $u=1-e+(1-e) \sigma_{i}(e) x_{i}$ and $v=e+(e-1) \sigma_{i}(e) x_{i}$. Then

$$
\begin{aligned}
u v= & e+(e-1) \sigma_{i}(e) x_{i}-e^{2}-e(e-1) \sigma_{i}(e) x_{i}+(1-e) \sigma_{i}(e) x_{i} e \\
& +(1-e) \sigma_{i}(e) x_{i}(e-1) \sigma_{i}(e) x_{i} \\
= & e \sigma_{i}(e) x_{i}-\sigma_{i}(e) x_{i}-e \sigma_{i}(e) x_{i}+e \sigma_{i}(e) x_{i}+(1-e) \sigma_{i}(e)\left(\sigma_{i}(e) x_{i}+\delta_{i}(e)\right) \\
& +(1-e) \sigma_{i}(e)\left(\sigma_{i}(e) x_{i}-x_{i}+\delta_{i}(e)\right) \sigma_{i}(e) x_{i} \\
= & -\sigma_{i}(e) x_{i}+e \sigma_{i}(e) x_{i}+\sigma_{i}(e) x_{i}+\sigma_{i}(e) \delta_{i}(e)-e \sigma_{i}(e) x_{i}-e \sigma_{i}(e) \delta_{i}(e) \\
& +\left[\sigma_{i}(e) x_{i}-\sigma_{i}(e) x_{i}+\sigma_{i}(e) \delta_{i}(e)-e \sigma_{i}(e) x_{i}+e \sigma_{i}(e) x_{i}-e \sigma_{i}(e) \delta_{i}(e)\right] \sigma_{i}(e) x_{i} \\
= & 0
\end{aligned}
$$

Hence, by the Armendariz condition, $(1-e)(e-1) \sigma_{i}(e)=0$, i.e., $e \sigma_{i}(e)=\sigma_{i}(e)$.

Now, let $w=e+e\left(1-\sigma_{i}(e)\right) x_{i}, z=1-e-e\left(1-\sigma_{i}(e)\right) x_{i}$ be elements of $A$. Then

$$
\begin{aligned}
w z= & e-e^{2}-e^{2}\left(1-\sigma_{i}(e)\right) x_{i}+e\left(1-\sigma_{i}(e)\right) x_{i}-e\left(1-\sigma_{i}(e)\right) x_{i} e \\
& -e\left(1-\sigma_{i}(e)\right) x_{i} e\left(1-\sigma_{i}(e)\right) x_{i} \\
= & -e\left(1-\sigma_{i}(e)\right) x_{i} e\left[1+\left(1-\sigma_{i}(e)\right) x_{i}\right] \\
= & -e\left(1-\sigma_{i}(e)\right)\left(\sigma_{i}(e) x_{i}+\delta_{i}(e)\right)\left[1+\left(1-\sigma_{i}(e)\right) x_{i}\right] \\
= & \left(-e \sigma_{i}(e) x_{i}+e \sigma_{i}(e) x_{i}\right)\left[1+\left(1-\sigma_{i}(e)\right) x_{i}\right] \\
= & 0
\end{aligned}
$$

since $\delta_{i}(e)=0$ and $\sigma_{i}(e) \sigma_{i}(e)=\sigma_{i}(e)$. Then, Armendariz condition implies $e(-e(1-$ $\left.\left.\sigma_{i}(e)\right)\right)=0$, which shows that $e \sigma_{i}(e)=e$, and so $\sigma_{i}(e)=e$. Therefore, we conclude the proof.

Next, we show that every weak $\Sigma$-skew Armendariz ring is Abelian.

Proposition 3.9. If $R$ is a weak $\Sigma$-skew Armendariz ring, then $R$ is Abelian.

Proof. Let $e^{2}=e, a \in R$. Consider the elements $f, g$ of $A$ given by $f=e-\sum_{i=1}^{n} e a(1-e) x_{i}$, and $g=1-e+\sum_{i=1}^{n} e a(1-e) x_{i}$. Since

$$
\begin{aligned}
f g= & e-e^{2}+e^{2} a(1-e) \sum_{i=1}^{n} x_{i}-\sum_{i=1}^{n} e a(1-e) x_{i}+\sum_{i=1}^{n} e a(1-e) x_{i} e \\
& -\left(\sum_{i=1}^{n} e a(1-e) x_{i} e a(1-e)\right)\left(\sum_{i=1}^{n} x_{i}\right) \\
= & \sum_{i=1}^{n} e a(1-e)\left(\sigma_{i}(e) x_{i}+\delta_{i}(e)\right)-\left(\sum_{i=1}^{n} e a(1-e) x_{i}(e a-e a e)\right)\left(\sum_{i=1}^{n} x_{i}\right) .
\end{aligned}
$$

[Revista Integración 
Equivalently,

$$
\begin{aligned}
f g= & (e a-e a e) \sum_{i=1}^{n} \sigma_{i}(e) x_{i} \\
& -\left(\sum_{i=1}^{n}(e a-e a e)\left(\sigma_{i}(e a-e a e) x_{i}+\delta_{i}(e a-e a e)\right)\right)\left(\sum_{i=1}^{n} x_{i}\right) \\
= & (e a-e a e) \sum_{i=1}^{n} \sigma_{i}(e) x_{i} \\
& -\left(\sum_{i=1}^{n}(e a-e a e)\left(\left(e \sigma_{i}(a)-e \sigma_{i}(a) e\right) x_{i}+\delta_{i}(e a)-\delta_{i}(e a e)\right)\right)\left(\sum_{i=1}^{n} x_{i}\right) \\
= & e a \sum_{i=1}^{n} \sigma_{i}(e) x_{i}-e a e \sum_{i=1}^{n} \sigma_{i}(e) x_{i} \\
& -\left(\sum _ { i = 1 } ^ { n } ( e a - e a e ) \left(e \sigma_{i}(a) x_{i}-e \sigma_{i}(a) e x_{i}+\sigma_{i}(e) \delta_{i}(a)\right.\right. \\
& \left.\left.-\sigma_{i}(e a) \delta_{i}(e)-\delta_{i}(e a) e\right)\right)\left(\sum_{i=1}^{n} x_{i}\right)
\end{aligned}
$$

or what is the same,

$$
\begin{aligned}
f g= & e a \sum_{i=1}^{n} \sigma_{i}(e) x_{i}-e a e \sum_{i=1}^{n} \sigma_{i}(e) x_{i} \\
& -\left(\sum_{i=1}^{n}(e a-e a e)\left(e \sigma_{i}(a) x_{i}-e \sigma_{i}(a) e x_{i}+e \delta_{i}(a)-e \delta_{i}(a) e\right)\right)\left(\sum_{i=1}^{n} x_{i}\right) \\
= & e a \sum_{i=1}^{n} \sigma_{i}(e) x_{i}-e a e \sum_{i=1}^{n} \sigma_{i}(e) x_{i}-\left(\sum _ { i = 1 } ^ { n } \left\{e a e \sigma_{i}(a) x_{i}-e a e \sigma_{i}(a) e x_{i}+e a e \delta_{i}(a)\right.\right. \\
& \left.\left.-e a e \delta_{i}(a) e-e a e \sigma_{i}(a) x_{i}+e a e \sigma_{i}(a) e x_{i}-e a e \delta_{i}(a)+e a e \delta_{i}(a) e\right\}\right)\left(\sum_{i=1}^{n} x_{i}\right) \\
= & 0 .
\end{aligned}
$$

Since $R$ is weak $\Sigma$-skew Armendariz, eea $(1-e)=0$, that is, ea $=e a e$. Now, consider the elements $p, q$ of $A$ given by $p=1-e-\sum_{i=1}^{n}(1-e) a e x_{i}$ and $q=e+\sum_{i=1}^{n}(1-e) a e x_{i}$. Then $p q=0$. More exactly,

$$
\begin{aligned}
p q= & e+\sum_{i=1}^{n}(1-e) a e x_{i}-e^{2}-e \sum_{i=1}^{n}(1-e) a e x_{i}-\sum_{i=1}^{n}(1-e) a e x_{i} e \\
& -\left(\sum_{i=1}^{n}(1-e) a e x_{i}\right)\left(\sum_{i=1}^{n}(1-e) \text { aex }_{i}\right)
\end{aligned}
$$

Vol. 34, No. 2, 2016] 
or what is the same,

$$
\begin{aligned}
p q= & \sum_{i=1}^{n}(1-e) a e x_{i}-\sum_{i=1}^{n}(1-e) a e\left(\sigma_{i}(e) x_{i}+\delta_{i}(e)\right) \\
& -\left(\sum_{i=1}^{n}(1-e) a e x_{i}(1-e) a e\right)\left(\sum_{i=1}^{n} x_{i}\right) \\
= & -\left(\sum_{i=1}^{n}(a e-e a e)\left(\sigma_{i}(a e-e a e) x_{i}+\delta_{i}(a e-e a e)\right)\right)\left(\sum_{i=1}^{n} x_{i}\right) \\
= & -\left(\sum_{i=1}^{n}(a e-e a e)\left(\sigma_{i}(a) e x_{i}-e \sigma_{i}(a) e x_{i}+\delta_{i}(a) e-\delta_{i}(e a) e\right)\right)\left(\sum_{i=1}^{n} x_{i}\right) \\
= & -\left(\sum _ { i = 1 } ^ { n } \left\{a e \sigma_{i}(a) e x_{i}-a e \sigma_{i}(a) e x_{i}+a e \delta_{i}(a) e-a e \sigma_{i}(e) \delta_{i}(a) e-e a e \sigma_{i}(a) e x_{i}\right.\right. \\
& \left.\left.+e a e \sigma_{i}(a) e x_{i}-e a e \delta_{i}(a) e+e a e \sigma_{i}(e) \delta_{i}(a) e\right\}\right)\left(\sum_{i=1}^{n} x_{i}\right) \\
= & 0
\end{aligned}
$$

since $e \sigma_{i}(e)=\sigma_{i}(e)=e$. By the weak $\Sigma$-skew Armendariz condition on $R$, we know that $(1-e)(1-e) a e=0$, or equivalently, ae =eae. Now, as it was shown above, ea =eae, which means that $a e=e a$, i.e., $R$ is Abelian.

Propositions $3.7,3.8$, and 3.9 imply the following corollary.

Corollary 3.10. If $R$ is a $\Sigma$-skew Armendariz ring $R$, then $A$ is an Abelian ring.

\section{Classical ring of quotients}

In this section we will characterize the Armendariz property for the classical ring of quotients of a bijective skew PBW extension. The key concept is the weak $\Sigma$-skew Armendariz property. Theorem 4.2 extends [24], Theorem 2.3, and [17], Theorem 16, and Theorem 4.3 generalizes [24], Corollary 2.5.

Let us recall the key facts about noncommutative localization. If $B$ is a ring and $S$ is a multiplicative subset of $B\left(1 \in S, 0 \notin S, s s^{\prime} \in S\right.$ for every $\left.s, s^{\prime} \in S\right)$, then the left ring of fractions of $B$ exists if and only if two conditions hold: (i) given $a \in B$ and $s \in S$ with $a s=0$, there exists $s^{\prime} \in S$ such that $s^{\prime} a=0$; (ii) (left Ore condition) given $a \in B$ and $s \in S$, there exist $s^{\prime} \in S$ and $a^{\prime} \in B$ with $s^{\prime} a=a^{\prime} s$. If these conditions hold, then the left ring of fractions of $B$ with respect to $S$ is denoted by $S^{-1} B$, and its elements are classes denoted using fractions. More exactly, $\frac{a}{s}:=\frac{b}{t}$ are equal if and only if there exist $c, d \in B$ such that $c a=d b, c s=d t \in S ; \frac{a}{s}+\frac{b}{t}:=\frac{c a+d b}{u}$, where $u:=c s=d t \in S$, for some $c, d \in B ; \frac{a}{s} \frac{b}{t}:=\frac{c b}{u s}$, where $u a=c t$, for some $u \in S$ and $c \in B$. Similarly, it is defined the right Ore condition, and hence the ring of fractions of $B$. The nonzero divisors elements of $B$ are called regular and the set of regular elements of $B$ is denoted by $S_{0}(B)$. Recall that if $B$ is both left and right Ore, then its classical left ring of quotients $Q_{c l}^{l}(B)$ and its 
classical right ring of quotients $Q_{c l}^{r}(B)$ coincide, and it is denoted by $Q(B)$. A key result about the classical ring of quotients of $B$ is the common denominator property: if $B$ is a ring, $S \subset B$ is a multiplicative subset and $S^{-1} B$ exists, then any finite set $\left\{q_{1}, \ldots, q_{n}\right\}$ of elements of $S^{-1} B$ posses a common denominator, i.e., there exist $r_{1}, \ldots, r_{n} \in B$ and $s \in S$ such that $q_{i}=\frac{r_{i}}{s}$ for every $i$ (see [15] for a detailed treatment of localization in noncommutative rings).

Proposition 4.1 ([21], Lemma 2.6). Let $A$ be a bijective skew PBW extension of a ring $R$. If $S \subseteq S_{0}(R)$ is a multiplicative subset of $R$ with $\sigma_{i}(S)=S$ for every $i=1, \ldots, n$, then

(a) If $S^{-1} R$ exists, then $S^{-1} A$ exists and it is a bijective skew $P B W$ extension of $S^{-1} R$, denoted $S^{-1} A=\sigma\left(S^{-1} R\right)\left\langle x_{1}^{\prime}, \ldots, x_{n}^{\prime}\right\rangle$, where $x_{i}^{\prime}:=\frac{x_{i}}{1}$, and the systems of constants of $S^{-1} R$ is given by $c_{i, j}^{\prime}=\frac{c_{i, j}}{1}, c_{i, \frac{r}{s}}^{\prime}:=\frac{\sigma_{i}(r)}{\sigma_{i}(s)}$, for $1 \leq i, j \leq n$. The automorphisms $\overline{\sigma_{i}}$ of $S^{-1} R$ and the $\overline{\sigma_{i}}$-derivations $\overline{\delta_{i}}(1 \leq i \leq n)$, are defined by $\overline{\sigma_{i}}\left(\frac{a}{s}\right):=\frac{\sigma_{i}(a)}{\sigma_{i}(s)}$, and $\overline{\delta_{i}}\left(\frac{a}{s}\right):=-\frac{\delta_{i}(s)}{\sigma_{i}(s)} \frac{a}{s}+\frac{\delta_{i}(a)}{\sigma_{i}(s)}$. Let $\bar{\Sigma}:=\left\{\overline{\sigma_{1}}, \ldots, \overline{\sigma_{n}}\right\}$ and $\bar{\Delta}:=\left\{\overline{\delta_{1}}, \ldots, \overline{\delta_{n}}\right\}$.

(b) If $R S^{-1}$ exists, then $A S^{-1}$ exists and it is a bijective skew $P B W$ extension of $R S^{-1}$, denoted $A S^{-1}=\sigma\left(R S^{-1}\right)\left\langle x_{1}^{\prime \prime}, \ldots, x_{n}^{\prime \prime}\right\rangle$, where $x_{i}^{\prime \prime}:=\frac{x_{i}}{1}$, and the systems of constants of $S^{-1} R$ is given by $c_{i, j}^{\prime \prime}=\frac{c_{i, j}}{1}, c_{i, \frac{r}{s}}^{\prime \prime}:=\frac{\sigma_{i}(r)}{\sigma_{i}(s)}$, for $1 \leq i, j \leq n$. The automorphisms $\overline{\sigma_{i}}$ of $S^{-1} R$ and the $\overline{\sigma_{i}}$-derivations $\overline{\delta_{i}}(1 \leq i \leq n)$, are defined by $\overline{\sigma_{i}}\left(\frac{a}{s}\right):=\frac{\sigma_{i}(a)}{\sigma_{i}(s)}$, and $\overline{\delta_{i}}\left(\frac{a}{s}\right):=-\frac{\sigma_{i}(a)}{\sigma_{i}(s)} \frac{\delta_{i}(s)}{s}+\frac{\delta_{i}(a)}{s}$. Let $\bar{\Sigma}:=\left\{\overline{\sigma_{1}}, \ldots, \overline{\sigma_{n}}\right\}$ and $\bar{\Delta}:=\left\{\overline{\delta_{1}}, \ldots, \overline{\delta_{n}}\right\}$.

If no confusion arises, we simply denote $x_{i}^{\prime}$ and $x_{i}^{\prime \prime}$ by $x_{i}$ for $1 \leq i \leq n$. Now, analogously to the definitions of $\Sigma$-rigid, $\Sigma$-skew Armendariz and weak $\Sigma$-skew Armendariz, we consider these notions, i.e., $\bar{\Sigma}$-rigid, $\bar{\Sigma}$-skew Armendariz and weak $\bar{\Sigma}$-skew Armendariz, for the classical quotient ring $Q(R)$ of $R$.

Next, Theorem 4.2 generalizes [24], Theorem 2.3, for Ore extensions of automorphism type, and hence, [17], Theorem 16, for reduced rings.

Theorem 4.2. Let $A$ be a bijective skew $P B W$ extension of a ring $R$. If the classical ring of quotients $Q(R)$ of $R$ exists, then $R$ is weak $\Sigma$-skew Armendariz if and only if $Q(R)$ is weak $\bar{\Sigma}$-skew Armendariz.

Proof. It is clear that if $Q(R)$ is weak $\bar{\Sigma}$-skew Armendariz, then $R$ is weak $\Sigma$-skew Armendariz.

Conversely, consider $f=c_{0}^{-1} a_{0}+\sum_{i=1}^{n} c_{i}^{-1} a_{i} x_{i}$ and $g=s_{0}^{-1} b_{0}+\sum_{j=1}^{n} s_{j}^{-1} b_{j} x_{j}$ elements of $S^{-1} A$ such that $f g=0$. Let us prove that $c_{i}^{-1} a_{i} \overline{\sigma_{i}}\left(s_{j}^{-1} b_{j}\right)=0$ for $0 \leq i, j \leq n$, where $\overline{\sigma_{0}}=\operatorname{id}_{S^{-1} A}$.

We know that there exist $a_{i}^{\prime}, b_{j}^{\prime} \in R$ and $c, s \in S_{0}(R)$ satisfying $c_{i}^{-1} a_{i}=c^{-1} a_{i}^{\prime}$ and

Vol. 34, No. 2, 2016] 
$s_{j}^{-1} b_{j}=s^{-1} b_{j}^{\prime}$ for $0 \leq i, j \leq n$. In this way, we can write

$$
\begin{aligned}
0 & =\left(c^{-1} a_{0}^{\prime}+\sum_{i=1}^{n} c^{-1} a_{i}^{\prime} x_{i}\right)\left(s^{-1} b_{0}^{\prime}+\sum_{j=1}^{n} s^{-1} b_{j}^{\prime} x_{j}\right) \\
& =\left(a_{0}^{\prime}+\sum_{i=1}^{n} a_{i}^{\prime} x_{i}\right) s^{-1}\left(b_{0}^{\prime}+\sum_{j=1}^{n} b_{j}^{\prime} x_{j}\right) \\
& =\left(a_{0}^{\prime} s^{-1}+\sum_{i=1}^{n} a_{i}^{\prime}\left[\overline{\sigma_{i}}\left(s^{-1}\right) x_{i}+\overline{\delta_{i}}\left(s^{-1}\right)\right]\right)\left(b_{0}^{\prime}+\sum_{j=1}^{n} b_{j}^{\prime} x_{j}\right) \\
& =\left(a_{0}^{\prime} s^{-1}+\sum_{i=1}^{n}\left(a_{i}^{\prime} \sigma_{i}(s)^{-1} x_{i}-a_{i}^{\prime} \sigma_{i}(s)^{-1} \delta_{i}(s) s^{-1}\right)\right)\left(b_{0}^{\prime}+\sum_{j=1}^{n} b_{j}^{\prime} x_{j}\right) .
\end{aligned}
$$

There exist $d_{i} \in R(1 \leq i \leq n)$ and $s_{2} \in S_{0}(R)$ such that $\delta_{i}(s) s^{-1}=s_{2}^{-1} d_{i}$, which shows that

$$
0=\left(a_{0}^{\prime} s^{-1}+\sum_{i=1}^{n}\left(a_{i}^{\prime} \sigma_{i}(s)^{-1} x_{i}-a_{i}^{\prime} \sigma_{i}(s)^{-1} s_{2}^{-1} d_{i}\right)\right)\left(b_{0}^{\prime}+\sum_{j=1}^{n} b_{j}^{\prime} x_{j}\right) .
$$

Since there exist $a_{0}^{\prime \prime}, a_{i}^{\prime \prime}, a_{i}^{\prime \prime \prime} \in R(1 \leq i \leq n)$ and $s_{3}, s_{4}, s_{5} \in S_{0}(R)$ with $a_{0}^{\prime} s^{-1}=$ $s_{3}^{-1} a_{0}^{\prime \prime}, a_{i}^{\prime} \sigma_{i}(s)^{-1}=s_{4}^{-1} a_{i}^{\prime \prime}$, and $a_{i}^{\prime} \sigma_{i}(s)^{-1} s_{2}^{-1}=s_{5}^{-1} a_{i}^{\prime \prime \prime}$, we have

$$
0=\left(s_{3}^{-1} a_{0}^{\prime \prime}+\sum_{i=1}^{n}\left(s_{4}^{-1} a_{i}^{\prime \prime} x_{i}-s_{5}^{-1} a_{i}^{\prime \prime \prime} d_{i}\right)\right)\left(b_{0}^{\prime}+\sum_{j=1}^{n} b_{j}^{\prime} x_{j}\right)
$$

Again, there exist $d_{0}, d_{i}^{\prime}, d_{i}^{\prime \prime} \in R(1 \leq i \leq n)$ and $t \in S_{0}(R)$ with $s_{3}^{-1} a_{0}^{\prime \prime}=t^{-1} d_{0}, s_{4}^{-1} a_{i}^{\prime \prime}=$ $t^{-1} d_{i}^{\prime}$, and $s_{5}^{-1} a_{i}^{\prime \prime \prime}=t^{-1} d_{i}^{\prime \prime}$, which guarantees that

$$
\begin{aligned}
0 & =\left(t^{-1} d_{0}+\sum_{i=1}^{n}\left(t^{-1} d_{i}^{\prime} x_{i}-t^{-1} d_{i}^{\prime \prime} d_{i}\right)\right)\left(b_{0}^{\prime}+\sum_{j=1}^{n} b_{j}^{\prime} x_{j}\right) \\
& =t^{-1}\left(d_{0}+\sum_{i=1}^{n}\left(d_{i}^{\prime} x_{i}-d_{i}^{\prime \prime} d_{i}\right)\right)\left(b_{0}^{\prime}+\sum_{j=1}^{n} b_{j}^{\prime} x_{j}\right) \\
& =\left(d_{0}-\sum_{i=1}^{n} d_{i}^{\prime \prime} d_{i}+\sum_{i=1}^{n} d_{i}^{\prime} x_{i}\right)\left(b_{0}^{\prime}+\sum_{j=1}^{n} b_{j}^{\prime} x_{j}\right) .
\end{aligned}
$$

By the Armendariz condition on $R$, from (2) we obtain the relations given by

$$
\left(d_{0}-\sum_{i=1}^{n} d_{i}^{\prime \prime} d_{i}\right) b_{j}^{\prime}=0 \quad(0 \leq j \leq n), \text { and } \quad d_{i}^{\prime} \sigma_{i}\left(b_{j}^{\prime}\right)=0 \quad(1 \leq i \leq n) \quad(0 \leq j \leq n)
$$


From the reasoning above we have the following equivalences for expressions in (3):

$$
\begin{aligned}
\left(d_{0}-\sum_{i=1}^{n} d_{i}^{\prime \prime} d_{i}\right) b_{j}^{\prime}=0 & \Leftrightarrow t^{-1}\left(d_{0}-\sum_{i=1}^{n} d_{i}^{\prime \prime} d_{i}\right) b_{j}^{\prime}=0 \\
& \Leftrightarrow\left(t^{-1} d_{0}-\sum_{i=1}^{n} t^{-1} d_{i}^{\prime \prime} d_{i}\right) b_{j}^{\prime}=0 \\
& \Leftrightarrow\left(s_{3}^{-1} a_{0}^{\prime \prime}-\sum_{i=1}^{n} s_{5}^{-1} a_{i}^{\prime \prime \prime} d_{i}\right) b_{j}^{\prime}=0 \\
& \Leftrightarrow\left(a_{0}^{\prime} s^{-1}-\sum_{i=1}^{n} a_{i}^{\prime} \sigma_{i}(s)^{-1} s_{2}^{-1} d_{i}\right) b_{j}^{\prime}=0 \\
& \Leftrightarrow\left(a_{0}^{\prime} s^{-1}-\sum_{i=1}^{n} a_{i}^{\prime} \sigma_{i}(s)^{-1} \delta_{i}(s) s^{-1}\right) b_{j}^{\prime}=0 \\
& \Leftrightarrow\left(a_{0}^{\prime} s^{-1}+\sum_{i=1}^{n} a_{i}^{\prime} \overline{\delta_{i}}\left(s^{-1}\right)\right) b_{j}^{\prime}=0 \quad(0 \leq j \leq n),
\end{aligned}
$$

and

$$
\begin{aligned}
d_{i}^{\prime} \sigma_{i}\left(b_{j}^{\prime}\right)=0 & \Leftrightarrow t^{-1} d_{i}^{\prime} \sigma_{i}\left(b_{j}^{\prime}\right)=0 \Leftrightarrow s_{4}^{-1} a_{i}^{\prime \prime} \sigma_{i}\left(b_{j}^{\prime}\right)=0 \\
& \Leftrightarrow a_{i}^{\prime} \sigma_{i}(s)^{-1} \sigma_{i}\left(b_{j}^{\prime}\right)=0 \Leftrightarrow a_{i}^{\prime} \overline{\sigma_{i}}\left(s^{-1}\right) \sigma_{i}\left(b_{j}^{\prime}\right)=0 \\
& \Leftrightarrow a_{i}^{\prime} \overline{\sigma_{i}}\left(s^{-1}\right) \overline{\sigma_{i}}\left(b_{j}^{\prime}\right)=0 \quad(1 \leq i \leq n)(0 \leq j \leq n) \\
& \Leftrightarrow c^{-1} a_{i}^{\prime} \overline{\sigma_{i}}\left(s^{-1}\right) \overline{\sigma_{i}}\left(b_{j}^{\prime}\right)=0 \Leftrightarrow c_{i}^{-1} a_{i} \overline{\sigma_{i}}\left(s^{-1}\right) \overline{\sigma_{i}}\left(b_{j}^{\prime}\right)=0 \\
& \Leftrightarrow c_{i}^{-1} a_{i} \overline{\sigma_{i}}\left(s^{-1} b_{j}^{\prime}\right)=0 \Leftrightarrow c_{i}^{-1} a_{i} \overline{\sigma_{i}}\left(s_{j}^{-1} b_{j}\right)=0(1 \leq i \leq n)(0 \leq j \leq n) .
\end{aligned}
$$

Now, from (2) we have

$$
\begin{aligned}
0= & \left(d_{0}-\sum_{i=1}^{n} d_{i}^{\prime \prime} d_{i}\right) b_{0}^{\prime}+\left(d_{0}-\sum_{i=1}^{n} d_{i}^{\prime \prime} d_{i}\right)\left(\sum_{j=1}^{n} b_{j}^{\prime} x_{j}\right) \\
& +\left(\sum_{i=1}^{n} d_{i}^{\prime} x_{i}\right) b_{0}^{\prime}+\left(\sum_{i=1}^{n} d_{i}^{\prime} x_{i}\right)\left(\sum_{j=1}^{n} b_{j}^{\prime} x_{j}\right) \\
= & \left(d_{0}-\sum_{i=1}^{n} d_{i}^{\prime \prime} d_{i}\right) b_{0}^{\prime}+\left(d_{0}-\sum_{i=1}^{n} d_{i}^{\prime \prime} d_{i}\right)\left(\sum_{j=1}^{n} b_{j}^{\prime} x_{j}\right) \\
& +\sum_{i=1}^{n} d_{i}^{\prime}\left[\sigma_{i}\left(b_{0}^{\prime}\right) x_{i}+\delta_{i}\left(b_{0}^{\prime}\right)\right]+\left(\sum_{i=1}^{n} d_{i}^{\prime} x_{i}\right)\left(\sum_{j=1}^{n} b_{j}^{\prime} x_{j}\right) \\
= & \left(d_{0}-\sum_{i=1}^{n} d_{i}^{\prime \prime} d_{i}\right) b_{0}^{\prime}+\sum_{j=1}^{n}\left(d_{0}-\sum_{i=1}^{n} d_{i}^{\prime \prime} d_{i}\right) b_{j}^{\prime} x_{j}+\sum_{i=1}^{n} d_{i}^{\prime} \sigma_{i}\left(b_{0}^{\prime}\right) x_{i}+\sum_{i=1}^{n} d_{i}^{\prime} \delta_{i}\left(b_{0}^{\prime}\right) \\
& +\sum_{i=1}^{n} d_{i}^{\prime} x_{i} b_{i}^{\prime} x_{i}+\sum_{i, j \in\{1, \ldots, n\}, i \neq j} d_{i}^{\prime} x_{i} b_{j}^{\prime} x_{j},
\end{aligned}
$$

Vol. 34, No. 2, 2016] 
or equivalently,

$$
\begin{aligned}
0= & \left(d_{0}-\sum_{i=1}^{n} d_{i}^{\prime \prime} d_{i}\right) b_{0}^{\prime}+\sum_{i=1}^{n} d_{i}^{\prime} \delta_{i}\left(b_{0}^{\prime}\right)+\sum_{j=1}^{n}\left\{\left(d_{0}-\sum_{i=1}^{n} d_{i}^{\prime \prime} d_{i}\right) b_{j}^{\prime}+d_{j}^{\prime} \sigma_{j}\left(b_{0}^{\prime}\right)\right\} x_{j} \\
& +\sum_{i=1}^{n} d_{i}^{\prime}\left[\sigma_{i}\left(b_{i}^{\prime}\right) x_{i}+\delta_{i}\left(b_{i}^{\prime}\right)\right] x_{i}+\sum_{i, j \in\{1, \ldots, n\}, i \neq j} d_{i}^{\prime} x_{i} b_{j}^{\prime} x_{j} \\
= & \left(d_{0}-\sum_{i=1}^{n} d_{i}^{\prime \prime} d_{i}\right) b_{0}^{\prime}+\sum_{i=1}^{n} d_{i}^{\prime} \delta_{i}\left(b_{0}^{\prime}\right)+\sum_{j=1}^{n}\left\{\left(d_{0}-\sum_{i=1}^{n} d_{i}^{\prime \prime} d_{i}\right) b_{j}^{\prime}+d_{j}^{\prime} \sigma_{j}\left(b_{0}^{\prime}\right)\right\} x_{j} \\
& +\sum_{i=1}^{n} d_{i}^{\prime} \sigma_{i}\left(b_{i}^{\prime}\right) x_{i}^{2}+\sum_{i=1}^{n} d_{i}^{\prime} \delta_{i}\left(b_{i}^{\prime}\right) x_{i}+\sum_{i, j \in\{1, \ldots, n\}, i \neq j} d_{i}^{\prime} x_{i} b_{j}^{\prime} x_{j} \\
= & \left(d_{0}-\sum_{i=1}^{n} d_{i}^{\prime \prime} d_{i}\right) b_{0}^{\prime}+\sum_{i=1}^{n} d_{i}^{\prime} \delta_{i}\left(b_{0}^{\prime}\right)+\sum_{j=1}^{n}\left\{\left(d_{0}-\sum_{i=1}^{n} d_{i}^{\prime \prime} d_{i}\right) b_{j}^{\prime}+d_{j}^{\prime} \sigma_{j}\left(b_{0}^{\prime}\right)+d_{j}^{\prime} \delta_{j}\left(b_{j}^{\prime}\right)\right\} x_{j} \\
& +\sum_{i=1}^{n} d_{i}^{\prime} \sigma_{i}\left(b_{i}^{\prime}\right) x_{i}^{2}+\sum_{i, j \in\{1, \ldots, n\}, i \neq j} d_{i}^{\prime} x_{i} b_{j}^{\prime} x_{j} .
\end{aligned}
$$

With the purpose of computing the last sum in (7), consider the Remark 2.3 (iii). Then

$$
\begin{aligned}
0= & \left(d_{0}-\sum_{i=1}^{n} d_{i}^{\prime \prime} d_{i}\right) b_{0}^{\prime}+\sum_{i=1}^{n} d_{i}^{\prime} \delta_{i}\left(b_{0}^{\prime}\right) \\
& +\sum_{j=1}^{n}\left\{\left(d_{0}-\sum_{i=1}^{n} d_{i}^{\prime \prime} d_{i}\right) b_{j}^{\prime}+d_{j}^{\prime} \sigma_{j}\left(b_{0}^{\prime}\right)+d_{j}^{\prime} \delta_{j}\left(b_{j}^{\prime}\right)\right\} x_{j} \\
& +\sum_{i=1}^{n} d_{i}^{\prime} \sigma_{i}\left(b_{i}^{\prime}\right) x_{i}^{2} \\
& +\sum_{i, j \in\{1, \ldots, n\}, i \neq j}\left\{\left[d_{i}^{\prime} \sigma_{i}\left(b_{j}^{\prime}\right)+d_{j}^{\prime} \sigma_{j}\left(b_{i}^{\prime}\right) c_{i, j}\right] x_{i} x_{j}+\left[d_{j}^{\prime} \delta_{j}\left(b_{i}^{\prime}\right)+d_{j}^{\prime} \sigma_{j}\left(b_{i}^{\prime}\right) r_{i}^{(i, j)}\right] x_{i}\right. \\
& \left.+\left[d_{i}^{\prime} \delta_{i}\left(b_{j}^{\prime}\right)+d_{j}^{\prime} \sigma_{j}\left(b_{i}^{\prime}\right)\right] x_{j}+d_{j}^{\prime} \sigma_{j}\left(b_{i}^{\prime}\right) r^{(i, j)}+d_{j}^{\prime} \sigma_{j}\left(b_{i}^{\prime}\right) \sum_{k=1, k \neq i, j}^{n} r_{k}^{(i, j)} x_{k}\right\} .
\end{aligned}
$$

By (3), the expression (8) takes the form

$$
0=\sum_{i=1}^{n} d_{i}^{\prime} \delta_{i}\left(b_{0}^{\prime}\right)+\sum_{j=1}^{n} d_{j}^{\prime} \delta_{j}\left(b_{j}^{\prime}\right) x_{j}+\sum_{i, j \in\{1, \ldots, n\}, i \neq j}\left[d_{j}^{\prime} \delta_{j}\left(b_{i}^{\prime}\right) x_{i}+d_{i}^{\prime} \delta_{i}\left(b_{j}^{\prime}\right) x_{j}\right],
$$

or equivalently,

$$
0=\sum_{i=1}^{n} d_{i}^{\prime} \delta_{i}\left(b_{0}^{\prime}\right)+\sum_{i, j \in\{1, \ldots, n\}} d_{i}^{\prime} \delta_{i}\left(b_{j}^{\prime}\right) x_{j} .
$$

By degree relations, we have necessarily the equalities

$$
\sum_{i=1}^{n} d_{i}^{\prime} \delta_{i}\left(b_{0}^{\prime}\right)=\sum_{i=1}^{n} d_{i}^{\prime} \delta_{i}\left(b_{j}^{\prime}\right)=0 .
$$


Indeed, for the first sum in (9) we note that

$$
\begin{aligned}
\sum_{i=1}^{n} d_{i}^{\prime} \delta_{i}\left(b_{0}^{\prime}\right)=0 & \Leftrightarrow \sum_{i=1}^{n} t^{-1} d_{i}^{\prime} \delta_{i}\left(b_{0}^{\prime}\right)=0 \Leftrightarrow \sum_{i=1}^{n} s_{4}^{-1} a_{i}^{\prime \prime} \delta_{i}\left(b_{0}^{\prime}\right)=0 \\
& \Leftrightarrow \sum_{i=1}^{n} a_{i}^{\prime} \sigma_{i}(s)^{-1} \delta_{i}\left(b_{0}^{\prime}\right)=0 \Leftrightarrow \sum_{i=1}^{n} c^{-1} a_{i}^{\prime} \sigma_{i}(s)^{-1} \delta_{i}\left(b_{0}^{\prime}\right)=0 \\
& \Leftrightarrow \sum_{i=1}^{n} c_{i}^{-1} a_{i} \sigma_{i}(s)^{-1} \delta_{i}\left(b_{0}^{\prime}\right)=0 .
\end{aligned}
$$

Similarly, for the second sum in (9) we have

$$
\begin{aligned}
\sum_{i=1}^{n} d_{i}^{\prime} \delta_{i}\left(b_{j}^{\prime}\right)=0 & \Leftrightarrow \sum_{i=1}^{n} t^{-1} d_{i}^{\prime} \delta_{i}\left(b_{j}^{\prime}\right)=0 \Leftrightarrow \sum_{i=1}^{n} s_{4}^{-1} a_{i}^{\prime \prime} \delta_{i}\left(b_{j}^{\prime}\right)=0 \\
& \Leftrightarrow \sum_{i=1}^{n} a_{i} \sigma_{i}(s)^{-1} \delta_{i}\left(b_{j}^{\prime}\right)=0 \Leftrightarrow \sum_{i=1}^{n} a_{i} \overline{\sigma_{i}}\left(s^{-1}\right) \overline{\delta_{i}}\left(b_{j}^{\prime}\right)=0 \quad(0 \leq j \leq n) .
\end{aligned}
$$

Now, consider the elements $h=a_{0}^{\prime}+\sum_{i=1}^{n} a_{i}^{\prime} x_{i}$, and $k=s^{-1} b_{j}^{\prime}$ with $j=1, \ldots, n$. Then

$$
\begin{aligned}
h k & =a_{0}^{\prime} s^{-1} b_{j}^{\prime}+\sum_{i=1}^{n} a_{i}^{\prime} x_{i} s^{-1} b_{j}^{\prime} \\
& =a_{0}^{\prime} s^{-1} b_{j}^{\prime}+\sum_{i=1}^{n} a_{i}^{\prime}\left[\overline{\sigma_{i}}\left(s^{-1}\right) \overline{\sigma_{i}}\left(b_{j}^{\prime}\right) x_{i}+\overline{\delta_{i}}\left(b_{j}^{\prime}\right)\right] \\
& =a_{0} s^{-1} b_{j}^{\prime}+\sum_{i=1}^{n} a_{i}^{\prime} \overline{\sigma_{i}}\left(s^{-1}\right) \overline{\sigma_{i}}\left(b_{j}^{\prime}\right) x_{i}+\sum_{i=1}^{n} a_{i}^{\prime} \overline{\delta_{i}}\left(s^{-1} b_{j}^{\prime}\right) \\
& =a_{0} s^{-1} b_{j}^{\prime}+\sum_{i=1}^{n} a_{i}^{\prime} \overline{\sigma_{i}}\left(s^{-1}\right) \overline{\sigma_{i}}\left(b_{j}^{\prime}\right) x_{i}+\sum_{i=1}^{n} a_{i}^{\prime} \overline{\sigma_{i}}\left(s^{-1}\right) \overline{\delta_{i}}\left(b_{j}^{\prime}\right)+\sum_{i=1}^{n} a_{i}^{\prime} \overline{\delta_{i}}\left(s^{-1}\right) b_{j}^{\prime} .
\end{aligned}
$$

By (4), (5) and (11), we obtain

$$
h k=a_{0} s^{-1} b_{j}^{\prime}+\sum_{i=1}^{n} a_{i}^{\prime} \delta_{i}\left(s^{-1}\right) b_{j}^{\prime}=\left(a_{0}^{\prime} s^{-1}+\sum_{i=1}^{n} a_{i}^{\prime} \delta_{i}\left(s^{-1}\right)\right) b_{j}^{\prime}=0, \quad 1 \leq j \leq n .
$$

Since there exist $m_{j} \in R$ for every $j$, and $n \in S_{0}(R)$ such that $s^{-1} b_{j}^{\prime}=m_{j} n^{-1}$, then $\left(a_{0}^{\prime}+\sum_{i=1}^{n} a_{i}^{\prime} x_{i}\right) m_{j} n^{-1}=0$, whence $\left(a_{0}^{\prime}+\sum_{i=1}^{n} a_{i}^{\prime} x_{i}\right) m_{j}=0$. By the Armendariz condition on $R, a_{0}^{\prime} m_{j}=a_{i}^{\prime} \sigma_{i}\left(m_{j}\right)=0$ for every $i$ and $0 \leq j \leq n$. Note that $a_{0}^{\prime} m_{j}=0$ is equivalent to $a_{0}^{\prime} m_{j} n^{-1}=0$, that is, $a_{0}^{\prime} s^{-1} b_{j}^{\prime}=0$ for each $1 \leq j \leq n$. Since we have the equivalences

$$
a_{0}^{\prime} s^{-1} b_{j}^{\prime}=0 \Leftrightarrow c^{-1} a_{0}^{\prime} s_{j}^{-1} b_{j}=0 \Leftrightarrow c_{0}^{-1} a_{0} s_{j}^{-1} b_{j}=0, \quad 0 \leq j \leq n,
$$

expressions (6) and (14) show that the $\operatorname{ring} Q(R)$ of $R$ is weak $\Sigma$-skew Armendariz. $\square$

Vol. 34, No. 2, 2016] 
Note that every Ore domain is a $\Sigma$-skew Armendariz ring for every family of automorphisms $\Sigma$ and every family of $\Sigma$-derivations $\Delta$ of $R$. Theorem 4.3 generalizes [24], Corollary 2.5.

Theorem 4.3. If $R$ is a semiprime Goldie ring and $A$ is a bijective skew $P B W$ extension of $R$, then the following statements are equivalent:

(i) $R$ is $\Sigma$-rigid;

(ii) $R$ is $\Sigma$-skew Armendariz;

(iii) $R$ is weak $\Sigma$-skew Armendariz;

(iv) $Q(R)$ is $\bar{\Sigma}$-rigid;

(v) $Q(R)$ is $\bar{\Sigma}$-skew Armendariz;

(vi) $Q(R)$ is weak $\bar{\Sigma}$-skew Armendariz.

Proof. The implications (i) $\Rightarrow$ (ii) $\Rightarrow$ (iii) and (iv) $\Rightarrow(v) \Rightarrow$ (vi) are clear. Equivalence (iii) $\Leftrightarrow$ (vi) is the content of Theorem 4.2. (ii) $\Rightarrow$ (i): If $R$ is a $\Sigma$-skew Armendariz ring, then $R$ is weak $\Sigma$-skew Armendariz, and by Theorem 4.2 we know that $Q(R)$ is weak $\bar{\Sigma}$-skew Armendariz, that is, $Q(R)$ is Abelian by Proposition 3.9. In this way, $Q(R)$ is an Abelian semisimple ring, i.e., $Q(R)$ is reduced. If $a \overline{\sigma^{\alpha}}(a)=0$ for an element $a \in Q(R)$, then $\overline{\sigma^{\alpha}}(a) a=0$, since $Q(R)$ is reduced. Note that Lemma 3.3 implies $\overline{\sigma^{\alpha}}(a) \overline{\delta^{\alpha}}\left(\overline{\sigma^{\alpha}}(a)\right)=\overline{\delta^{\alpha}}(a) \overline{\sigma^{\alpha}}(a)=0$.

Consider the elements $h=\overline{\sigma^{\alpha}}(a)-\overline{\sigma^{\alpha}}(a) x^{\alpha}$ and $k=a+\overline{\sigma^{\alpha}}(a) x^{\alpha}$ of $\sigma(Q(R))\left\langle x_{1}, \ldots, x_{n}\right\rangle$. Then

$$
\begin{aligned}
h k & =\overline{\sigma^{\alpha}}(a) a+\left(\overline{\sigma^{\alpha}}(a)\right)^{2} x^{\alpha}-\overline{\sigma^{\alpha}}(a) x^{\alpha} a-\overline{\sigma^{\alpha}}(a) x^{\alpha} \overline{\sigma^{\alpha}}(a) x^{\alpha} \\
& =\left(\overline{\sigma^{\alpha}}(a)\right)^{2} x^{\alpha}-\overline{\sigma^{\alpha}}(a)\left[\overline{\sigma^{\alpha}}(a) x^{\alpha}+p_{\alpha, a}^{\prime}\right]-\overline{\sigma^{\alpha}}(a)\left[\overline{\sigma^{\alpha}}\left(\overline{\sigma^{\alpha}}(a)\right) x^{\alpha}+q_{\alpha, \overline{\sigma^{\alpha}}(a)}^{\prime}\right] x^{\alpha} \\
& =-\overline{\sigma^{\alpha}}(a) p_{\alpha, a}^{\prime}-\overline{\sigma^{\alpha}}\left(a \overline{\sigma^{\alpha}}(a)\right) x^{\alpha} x^{\alpha}-\overline{\sigma^{\alpha}}(a) q_{\alpha, \overline{\sigma^{\alpha}}(a)}^{\prime} x^{\alpha} \\
& =-\overline{\sigma^{\alpha}}(a) p_{\alpha, a}^{\prime}-\overline{\sigma^{\alpha}}(a) q_{\alpha, \bar{\sigma}^{\alpha}(a)}^{\prime} x^{\alpha},
\end{aligned}
$$

where the polynomials $p_{\alpha, a}^{\prime}$ and $q_{\alpha, \overline{\sigma^{\alpha}}(a)}^{\prime}$ have the properties established in Proposition 2.7. Since $a \overline{\sigma^{\alpha}}(a)=\overline{\sigma^{\alpha}}(a) a=0$, Remark 2.8 (ii) and Lemma 3.3 show that $\overline{\sigma^{\alpha}}(a) p_{\alpha, a}^{\prime}=$ $\overline{\sigma^{\alpha}}(a) q_{\alpha, \overline{\sigma^{\alpha}}(a)}^{\prime}=0$, whence $h k=0$. Since $Q(R)$ is weak $\bar{\Sigma}$-skew Armendariz, we have $\overline{\sigma^{\alpha}}(a) \overline{\sigma^{\alpha}}(a)=0$, and using the fact that $Q(R)$ is reduced and $\overline{\sigma^{\alpha}}$ is a monomorphism, we obtain $a=0$, that is, $Q(R)$ is $\bar{\Sigma}$-rigid, and so $R$ is $\Sigma$-rigid. With this reasoning we have proved also (vi) $\Rightarrow$ (iv) and (iii) $\Rightarrow$ (i).

\section{Baer, quasi-Baer, p.p. and p.q.-rings}

Kaplansky in [16] defined a ring $B$ as a Baer (resp. quasi-Baer, which was defined by Clark in [8]) ring if the right annihilator of every nonempty subset (resp. ideal) of $B$ is generated by an idempotent. Another generalization of Baer rings are the p.p.-rings. A ring $B$ is called right (resp. left) p.p if the right (resp. left) annihilator of each element 
of $B$ is generated by an idempotent (or equivalently, rings in which each principal right (resp. left) ideal is projective). Birkenmeier et al. in [6] define a ring to be called a right (resp. left) principally quasi-Baer (or simply right (resp. left) p.q-Baer) ring if the right annihilator of each principal right (resp. left) ideal of $B$ is generated by an idempotent.

Commutative and noncommutative rings Baer, quasi-Baer, p.p.-rings, and right p.q.Baer have been investigated in the literature. For instance, polynomial extensions in the commutative case were studied in [2], [4], and Ore extensions $B[x ; \sigma, \delta]$ of injective type, i.e., when $\sigma$ is injective, of all this kind of rings can be found in several works (cf. $[5],[6],[10],[13]$, and others). Some of these treatments consider the case $\delta=0$ and $\sigma$ an automorphism, or the case where $\sigma$ is the identity. Nevertheless, it is important to say that the Baerness and quasi-Baerness of a $\operatorname{ring} B$ do not inherite the Ore extension of $B$. More exactly, there are examples which show that there exists a Baer ring $B$ but the Ore extension $B[x ; \sigma, \delta]$ is not right p.q.-Baer ([10], Example 8); similarly, there exist Ore extensions $B[x ; \sigma, \delta]$ which are quasi-Baer, but $B$ is not quasi-Baer ([10], Example 9 and [3], Example 11). In general, the Baerness of $B$ and $B[x ; \sigma, \delta]$ does not depend on each other. Since Ore extensions of injective type are particular examples of skew PBW extensions, the concepts of Baer, quasi-Baer, and p.p. and p.q. are interesting for the ring theoretical study of skew PBW extensions. With this in mind, the first author in [28] studied all these concepts using the notion of $\Sigma$-rigid rings and established necessary and sufficient conditions to guarantee their stability under skew PBW extensions, generalizing the results presented in [13].

Now, as we saw in expression (1), $\Sigma$-rigid are contained strictly in weak $\Sigma$-skew Armendariz rings, so our next task is to consider all above concepts over these more general rings. With this objective, it is useful the following notation: for a nonempty subset $D$ of a ring $B$, we denote by $r_{B}(D)=\{b \in B \mid d b=0, \forall d \in D\}$, the right annihilator of $D$ in $B$.

Theorem 5.1. If $A$ is a bijective skew $P B W$ extension of a weak $\Sigma$-skew Armendariz ring $R$, then $R$ is a Baer ring if and only if $A$ is a Baer ring.

Proof. Suppose that $R$ is a Baer ring. Consider a nonempty subset $C$ of $A$ and let $C^{*}$ be the set of all coefficients of elements of $C$. It is clear that $C^{*}$ is a nonempty subset of $R$, and by assumption, $r_{R}\left(C^{*}\right)=e R$ for some idempotent $e \in R$. If $f=\sum_{k=0}^{m} a_{k} X_{k}$ is an element of $A$, Remark $2.8(\mathrm{i})$, and the equalities $\sigma_{i}(e)=e$ and $\delta_{i}(e)=0$ for $1 \leq i \leq n$ (Proposition 3.8), show that $X_{k} e=0$ for every $1 \leq k \leq m$, and so $f e=0$, that is, $e \in r_{A}(C)$, which implies $e A \subseteq r_{A}(C)$. Now, if $g=\sum_{j=0}^{t} b_{j} Y_{j}$ is a nonzero element of $r_{A}(C)$, then $f g=0$ for any $f \in C$. Since $R$ is $\Sigma$-skew Armendariz, $\sigma^{\alpha_{k}}\left(b_{j}\right) \in r_{R}\left(C^{*}\right)=$ $e R$ for every $1 \leq k \leq m$ with $\alpha_{k}=\exp \left(X_{k}\right)$, whence $b_{0}, b_{1}, \ldots, b_{t} \in e R$, since $\sigma^{\alpha_{k}}$ is an automorphism for every $k$ and $\sigma_{i}(e)=e$ for $1 \leq i \leq n$. Therefore, $g$ takes the form $g=\sum_{j=0}^{t} e d_{j}=e \sum_{j=1}^{t} d_{j} \in e A$ for some elements $d_{j} \in R$, which shows that $r_{A}(C) \subseteq e A$, and so $r_{A}(C)=e A$.

Conversely, if $A$ is Baer and $B$ is a nonempty subset of $R$, then $r_{A}(B)=e A$ for some idempotent $e \in R$ (Proposition 3.7). Using this fact, we obtain $r_{R}(B)=r_{A}(B) \cap R=$ $e A \cap R=e R$, that is, $R$ is Baer.

Corollary 5.2 ([28], Theorem 3.9). Let $R$ be a $\Sigma$-rigid ring. Then $R$ is a Baer ring if and only if $A$ is a Baer ring.

Vol. 34, No. 2, 2016] 
Theorem 5.3. If $A$ is a bijective skew $P B W$ extension of a weak $\Sigma$-skew Armendariz ring $R$, then $R$ is a p.p.-ring if and only if $A$ is a p.p.-ring.

Proof. Suppose that $R$ is a p.p.-ring and let $f=\sum_{k=0}^{m} a_{k} X_{k}$ a nonzero element of $A$. Then there exists an idempotent $e_{k} \in R$ such that $r_{R}\left(\left\{a_{k}\right\}\right)=e_{k} R$, for every $0 \leq k \leq m$. Let $e:=e_{0} e_{1} \cdots e_{m}$. It is clear that $e^{2}=e \in R$ and $e R=\bigcap_{k=0}^{m} r_{R}\left(\left\{a_{k}\right\}\right)$, since $R$ is Abelian (Proposition 3.9). By Proposition 3.8, we have $\sigma_{i}(e)=e$ and $\delta_{i}(e)=0$ for $1 \leq i \leq n$; so, using a reasoning as above, $f e=0$, which shows that $e A \subseteq r_{A}(\{f\})$. Let us see the another inclusion. If $g=\sum_{j=0}^{t} b_{j} X_{j} \in r_{A}(f)$, then $f g=0$, and by assumption, $a_{k} \sigma^{\alpha_{k}}\left(b_{j}\right)=0\left(\alpha_{k}=\exp \left(X_{k}\right)\right)$ whence $\sigma^{\alpha_{k}}\left(b_{j}\right) \in r_{R}\left(\left\{a_{k}\right\}\right)=e_{k} R$, for every $0 \leq k \leq m, 0 \leq j \leq t$. In this way, $b_{j} \in e_{k} R$, since $\sigma^{\alpha_{k}}$ is an automorphism with $\sigma_{i}(e)=e$, for every $1 \leq i \leq n$. Hence $b_{j} \in e R=\bigcap_{k=0}^{m} r_{R}\left(\left\{a_{k}\right\}\right)$, for all $0 \leq j \leq t$, which shows that $g \in e A$. Therefore $e A=r_{A}(\{f\})$, i.e., $A$ is a p.p.-ring.

Now, if $A$ is a p.p.-ring and $r$ is an element of $R$, we know that there exists an idempotent $e \in R$ with $r_{A}(\{a\})=e A$ (Proposition 3.7), which implies $r_{R}(\{a\})=e R$, and hence $R$ is a p.p.-ring.

Corollary 5.4 ([28], Theorem 3.12). Let $R$ be a $\Sigma$-rigid ring. Then $R$ is a p.p.-ring if and only if $A$ is a p.p.-ring.

Corollary 5.5 ([28], Theorems 3.10 and 3.13). Let $A$ be a bijective skew PBW extension of a reduced and weak $\Sigma$-skew Armendariz ring $R$. Then $R$ is a quasi-Baer (p.q.-Baer) ring if and only if $A$ is a quasi-Baer (p.q.-Baer) ring.

Remark 5.6. (i) ([11], Example 2.8). Let $B=\mathbb{k}[t]$ be the polynomial ring over a field $\mathbb{k}$ and $\sigma$ be the endomorphism given by $\sigma(f(t))=f(0)$. Then $B$ is quasi-Baer, but the ring $B[x ; \sigma]$ is not a quasi-Baer ring. This example shows that the injectivity of the endomorphisms $\sigma_{i} \in \Sigma$ (Proposition 2.2) is not a superfluous condition in Corollary 5.5.

(ii) Since prime rings are quasi-Baer, if $A$ is a bijective skew PBW extension of a prime ring $R$, then $A$ is prime ([27], Proposition 3.3 or [20], Corollary 4.2) and hence quasi-Baer.

(iii) In [28], Theorems $3.9,3.10,3.12$, and 3.13 the first author did not assume bijectivity on the injective endomorphisms $\sigma_{i}$ of $\Sigma$. More exactly, it was only required the invertibility of the elements $c_{i, j}$ in Definition 2.1. In this way, the results presented in [28] are valid for general skew PBW extensions satisfying these conditions on the elements $c_{i, j}$. Now, as we saw in this paper, $\Sigma$-rigid rings are contained strictly in weak $\Sigma$-skew Armendariz rings, so Theorems 5.1, 5.3, and Corollary 5.5 generalizes [28], Theorems 3.9, 3.10, 3.12, and 3.13. It is important to say that these general results are formulated assuming the bijectivity of the injective endomorphisms $\sigma_{i} \in \Sigma$. One could consider that this requirement is very strong; however, this is not the case. Indeed, several remarkable results about skew PBW extensions are formulated considering this hypothesis: the Hilbert basis theorem, regularity, Serre's Theorem, Global, Krull, Goldie and Gelfand-Kirillov dimensions, Quillen's $K$-groups, characterization of prime ideals, etc. (see [20], [22], [26], [29], and others). Last, but not least important, it is the number of examples of bijective skew PBW extensions which have been found in the literature (see [22] for a detailed list). 


\section{Acknowledgements}

The authors gratefully acknowledge the many helpful remarks of the referees. The first author would like to thank the Departamento de Matemáticas, Facultad de Ciencias, Universidad Nacional de Colombia for its financial support - Grant HERMES CODE 30366 -.

\section{References}

[1] Anderson D.D. and Camillo V., "Armendariz rings and Gaussian rings", Comm. Algebra 26 (1998), No. 7, 2265-2272.

[2] Armendariz E.P., "A note on extensions of Baer and p.p.-rings", J. Aust. Math. Soc. 18, (1974), 470-473.

[3] Armendariz E.P., Koo H.K. and Park J.K., "Isomorphic Ore extensions", Comm. Algebra 15 (1987), No. 12, 2633-2652.

[4] Birkenmeier G.F., "Baer rings and quasi-continuous rings have a MDSN", Pacific J. Math. 97 (1981), No. 2, 283-292.

[5] Birkenmeier G.F., Kim J.Y. and Park J.K., "Polynomial extensions of Baer and quasi-Baer rings", J. Pure Appl. Algebra 159 (2001), No. 1, 25-42.

[6] Birkenmeier G.F., Kim J.Y. and Park J.K., "Principally quasi-Baer rings", Comm. Algebra 29 (2001), No. 2, 639-660.

[7] Chen W. and Tong W., "A note on skew Armendariz rings", Comm. Algebra 33 (2005), No. 4, 1137-1140.

[8] Clark W.E., "Twisted matrix units semigroup algebras", Duke Math. J. 34 (1967), 417-423.

[9] Gallego C. and Lezama O., "Gröbner bases for ideals of $\sigma$-PBW extensions", Comm. Algebra 39 (2011), No. 1, 50-75.

[10] Han J., Hirano Y. and Kim H., "Semiprime Ore extensions", Comm. Algebra 28 (2000), No. 8, 3795-3801.

[11] Han J., Hirano Y. and Kim H., "Some results on skew polynomial rings over a reduced ring", in International Symposium on Ring Theory (Kyongju, 1999), Trends Math., Birkhäuser Boston, Boston, MA, (2001), 123-129.

[12] Hong C.Y., Kim N.K. and Kwak T.K., "On skew Armendariz rings", Comm. Algebra 31 (2003), No. 1, 103-122.

[13] Hong C.Y., Kim N.K. and Kwak T.K., "Ore extensions of Baer and p.p.-rings", J. Pure Appl. Algebra 151 (2000), No. 3, 215-226.

[14] Huh C., Lee Y. and Smoktunowicz A., "Armendariz rings and semicommutative rings", Comm. Algebra 30 (2002), No. 2, 751-761.

[15] Jategaonkar A.V., Localization in Noetherian rings, London Mathematical Society Lecture Note Series, 98, Cambridge University Press, Cambridge, 1986.

[16] Kaplansky I., Rings of operators. W.A. Benjamin, Inc., New York-Amsterdam, 1968.

Vol. 34, No. 2, 2016] 
[17] Kim N.K. and Lee Y., "Armendariz rings and reduced rings", J. Algebra 223 (2000), No. 2, 477-488.

[18] Krempa J., "Some examples of reduced rings", Algebra Colloq. 3 (1996), No. 4, 289-300.

[19] Lee T-K. and Wong T-L., "On Armendariz rings", Houston J. Math. 29 (2003), No. 3, 583-593.

[20] Lezama O., Acosta J.P. and Reyes A., "Prime ideals of skew PBW extensions", Rev. Un. Mat. Argentina 56 (2015), No. 2, 39-55.

[21] Lezama O., Acosta J.P., Chaparro C., Ojeda I. and Venegas C., "Ore and Goldie theorems for skew PBW extensions", Asian-Eur. J. Math. 6 (2013), No. 4, 20 pp.

[22] Lezama O. and Reyes A., "Some homological properties of skew PBW extensions", Comm. Algebra 42 (2014), No. 3, 1200-1230.

[23] Matczuk J., "A characterization of $\sigma$-rigid rings", Comm. Algebra 32 (2004), No. 11, 43334336.

[24] Nasr-Isfahani A.R. and Moussavi A., "On classical quotient rings of skew Armendariz rings", Int. J. Math. Math. Sci. (2007), 7 pp.

[25] Rege M.B. and Chhawchharia S., "Armendariz rings", Proc. Japan Acad. Ser. A Math. Sci. 73 (1997), No. 1, 14-17.

[26] Reyes A., "Gelfand-Kirillov dimension of skew PBW extensions", Rev. Colombiana Mat. 47 (2013), No. 1, 95-111.

[27] Reyes A., "Jacobson's conjecture and skew PBW extensions", Rev. Integr. Temas Mat. 32 (2014), No. 2, 139-152.

[28] Reyes A., "Skew PBW extensions of Baer, quasi-Baer, p.p. and p.q.-rings", Rev. Integr. Temas Mat. 33 (2015), No. 2, 173-189.

[29] Reyes A., "Uniform dimension over skew PBW extensions", Rev. Colombiana Mat. 48 (2014), No. 1, 79-96.

[30] Suárez H., Lezama O. and Reyes A., "Some Relations between N-Koszul, Artin-Schelter Regular and Calabi-Yau algebras with Skew PBW Extensions", Ciencia en Desarrollo 6 (2015), No. 2, 205-213. 